\title{
Disposable microfluidic devices: fabrication, function, and application
}

\author{
Gina S. Fiorini and Daniel T. Chiu
}

BioTechniques 38:429-446 (March 2005)

\begin{abstract}
This review article describes recent developments in microfluidics, with special emphasis on disposable plastic devices. Included is an overview of the common methods used in the fabrication of polymer microfluidic systems, including replica and injection molding, embossing, and laser ablation. Also described are the different methods by which on-chip operations-such as the pumping and valving of fluid flow, the mixing of different reagents, and the separation and detection of different chemical species-have been implemented in a microfluidic format. Finally, a few select biotechnological applications of microfluidics are presented to illustrate both the utility of this technology and its potential for development in the future.
\end{abstract}

\section{INTRODUCTION}

Similar to advances with integrated circuits in the computer industry during the past few decades, the area of biological and chemical analysis is also undergoing a miniaturization effort. Microfluidics, also referred to as lab-on-a-chip or micrototal analysis systems ( $\mu \mathrm{TAS}$ ), has seen great progress in the last decade and offers promising potential in the future. The premise of microfluidics is the manipulation and analysis of fluids within micrometersized channels, which, due to the small size, must be microfabricated using techniques adapted from the semiconductor and plastics industries, such as micromachining, photolithography, replica molding, embossing, and injection molding. Analogous to the benefits associated with miniaturization in the integrated circuits industry, the benefits associated with miniaturization of bioanalytical techniques include the reduction of the size of equipment, fast analysis and short reaction times, parallel operation for multiple analyses, and the possibility of portable devices. Specific to the field of microfluidics is the benefit of low sample and reagent volumes, leading to low waste levels and the unique physical consequences of microscale fluid flow.

A major goal of microfluidics research is the implementation and integration of various fluid manipulation components (e.g., pumps, valves, filters, and mixers) and analytical separation and detection techniques (e.g., electrophoresis, chromatography, fluorescence, and electrochemical detection) on individual microfabricated devices for complete on-chip analysis. To date, microfluidics has been successfully implemented in a variety of biological applications, including DNA sequencing and fragment sizing, PCR amplification, amino acid, peptide, and protein analysis, immunoassays, cell sorting and manipulation, and in vitro fertilization.

Given the scope of the field of microfluidics, this review is intended to provide an overview of techniques in microfluidics that are relevant to biological analysis and is not meant to be a comprehensive treatment of the research in this area. In particular, we will focus on disposable microfluidic devices and discuss common methods of fabrication, on-chip functions, and their usage in bioanalytical applica- tions. We refer readers to other review articles for more in-depth coverage of specific areas (1-3); each section below also contains references to appropriate topical reviews.

\section{OVERVIEW OF MICRO- FABRICATION METHODS}

A variety of methods exist for the fabrication of microfluidic devices, including wet etching, reactive ion etching, conventional machining (4), photolithography, soft lithography, hot embossing, injection molding, laser ablation, in situ construction (5), and plasma etching (6). In the following sections, we emphasize the most common methods used for the fabrication of disposable devices $(7,8)$ and will provide a brief description of the specific characteristics, advantages, and disadvantages of each method. As a general rule, the choice of fabrication method is determined by several factors, such as available technologies and equipment, cost, speed, fabrication capabilities (e.g., desired feature size and profile), and the preferred material substrate. 


\section{Micromachining of Silicon and Glass}

Early microfluidic devices were composed of silicon or glass (9-12) and were fabricated using micromachining techniques borrowed from the semiconductor industry. Micromachining of silicon and glass involves the use of wet and dry etching, photolithography, electron beam lithography, and a variety of other techniques, all of which require the use of cleanroom facilities and equipment. In the fabrication of disposable microfluidic systems, these micromachining steps are often used to generate the master from which disposable plastic devices are replicated (13-16).

Glass devices are commonly used in microfluidics due to the straightforward and well-understood fabrication techniques, as well as the beneficial optical properties, surface stability, and solvent compatibility of glass, and it is likely that glass devices will continue to be utilized in many applications in the years to come $(17,18)$. The high cost involved in processing glass and of the material itself, however, will likely limit their usage as disposable devices.

\section{Replica Molding}

Soft lithography: polydimethylsiloxane. A term coined by Whitesides and coworkers, soft lithography is a diverse set of techniques that encompasses replica molding using an elastomeric material, specifically polydimethylsiloxane (PDMS), for the fabrication of microfluidic devices as well as for the patterning of surfaces using PDMS stamps (microcontact printing) (19). The common procedure used for making PDMS microchannels involves the fabrication of a silicon master with patterned features composed of a photoresist (a photo-active polymer commonly used in photolithography, e.g., SU-8). In this process, silicon wafers are coated with photoresist and then exposed to ultraviolet (UV) light through a mask with the desired pattern (20-22). High-resolution transparencies can be used as the photomask to rapid prototype SU-8 patterned masters, from which PDMS molds can be replicated. Using transparency photomasks, the feature sizes are usually limited to $8 \mu \mathrm{m}$ and larger. For smaller feature sizes, a chrome mask, which can be substantially more expensive (approximately 100 times more expensive) than transparencies, is typically required. The photolithography process is easily repeated on wafers to produce multiple layers of patterned photoresist (i.e., multilayer features), and thin membranes of patterned PDMS can be stacked together to create multilayer 3-dimensional microfluidic systems (Figure 1A) $(23,24)$.

Replica molding with PDMS involves pouring PDMS prepolymer, a two-component mixture of base and cross-linking agent, directly onto a SU8 patterned silicon master and curing at a moderately elevated temperature $\left(60^{\circ} \mathrm{C}\right.$ for approximately $\left.2 \mathrm{~h}\right)$ to replicate the desired features (Figure 2A). To obtain an enclosed microchannel device, the PDMS replica can be conformally sealed (reversible) to a variety of materials, or the PDMS replica can be exposed to oxygen plasma prior to bonding (irreversible) to glass or another piece of PDMS (20). Soft lithography using PDMS offers several advantages, including low cost, fast processing $(<1$ day to final device), reusability of the SU-8 masters, facile sealing and bonding to a number of different substrates, and multilayer fabrication to create complex 3-dimensional systems. In addition to the benefits of the fabrication process, PDMS itself is suitable for a variety of biological and cellular applications $(19,25)$, due to the high gas permeability of PDMS. PDMS also has excellent optical transparency down to approximately $230 \mathrm{~nm}$. The elastomeric nature of PDMS makes possible easy installation of fluidic interconnects from the macro-world to the microfluidic device, as well as flexible on-chip components to control fluid flow (26-28). For certain applications, however, these same advantages may prove to be undesirable. For example, the gas permeability of PDMS is unsuitable for carrying out in-channel oxygen-sensitive polymerization reactions. Another drawback is the limited compatibility of PDMS with organic solvents, which tends to limit its use to aqueous solutions (29). A recent report on the synthesis of photo-

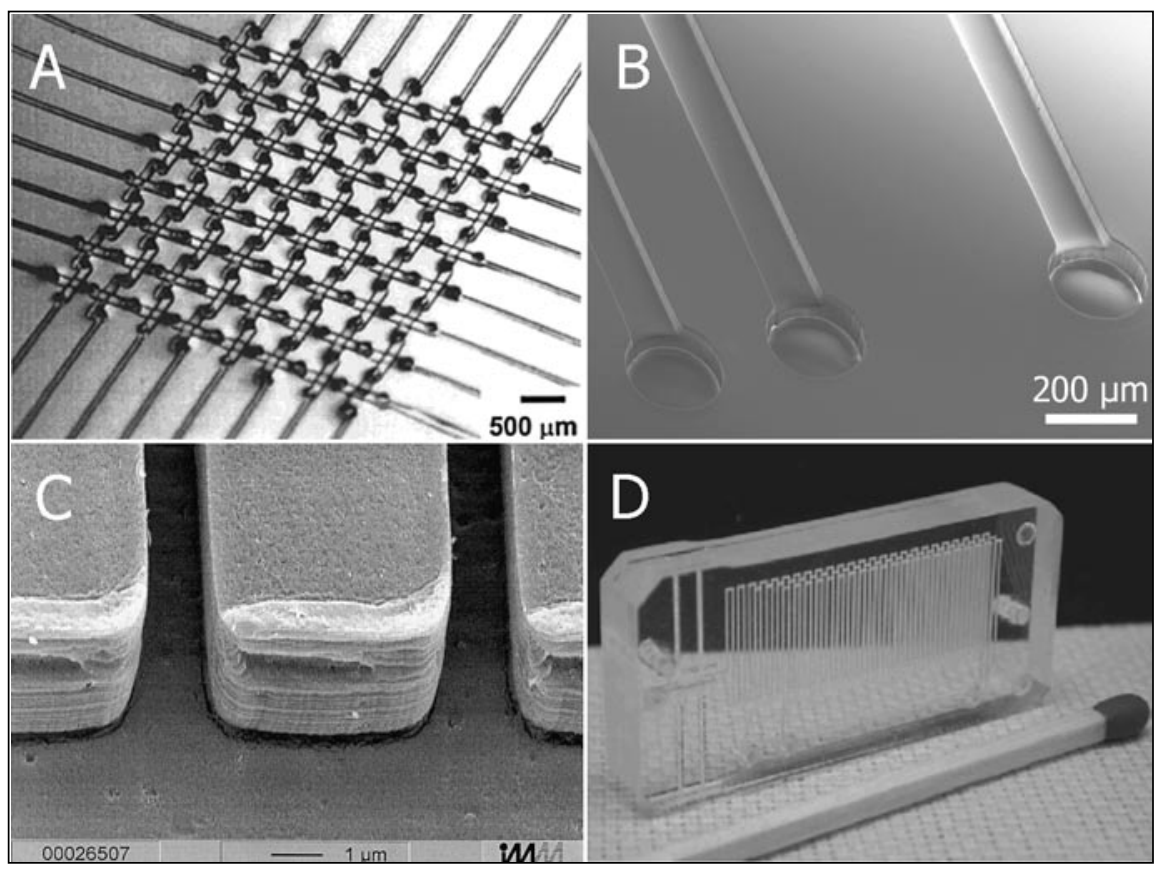

Figure 1. Disposable polymer microfluidic devices. (A) A 3-dimensional polydimethylsiloxane (PDMS) microchannel system in a basketweave pattern. (B) Multilayer microchannels replicated in thermoset polyester (TPE). (C) Microstructures embossed in polymethylmethacrylate (PMMA). (D) PMMA device fabricated using laser ablation $\left(\mathrm{CO}_{2}\right.$ laser). (A) Reprinted in part with permission from Reference 23, (C) 2000, American Chemical Society; (C) reprinted from Reference 33, () 2000, with permission from Elsevier; (D) reproduced from Reference 39 by permission of The Royal Society of Chemistry. 
curable perfluoropolyethers (PFPEs) with material properties and fabrication capabilities similar to PDMS may offer an alternative elastomer suitable for solvent-based applications (30).

Thermoset polyester. Replica molding using thermoset polyester (TPE) is accomplished in a manner similar to soft lithography with PDMS (Figure 2B) $(31,32)$. Pre-cured TPE resin is poured onto a SU-8 patterned silicon master, and curing of the material against the master yields the desired pattern. TPE differs from PDMS in that TPE is semi-cured first using UV light, removed from the master, and then allowed to completely cure against a second semi-cured TPE piece to obtain the final bonded channel (31).

TPE replica molding is inexpensive, fast, and capable of multilayer castings (Figure 1B), advantages stemming from the similarities to fabrication methods in soft lithography, and TPE has optical transparency (up to approximately $90 \%$ ) in the visible wavelength region, as well as compatibility with nonpolar solvents (e.g., cyclohexane, n-heptane, and toluene) that are unsuitable for use in PDMS devices. TPE, in contrast to PDMS, is not elastomeric, and when cured, is a hard material, and as such, requires fluidic interconnects different than those used with PDMS. In addition, TPE is not gas permeable, making TPE suitable for oxygen-sensitive applications, but probably unsuitable for longterm cell-based applications (e.g., cell culture). Overall, TPE replica molding is complementary to PDMS fabrication in that TPE fabrication utilizes the benefits of soft lithography-based techniques, yet offers different material properties.

\section{Embossing}

Embossing is a technique that involves the use of thermoplastic materials, typically in the form of flat sheets, which are patterned against a master (stamp) using pressure and heat (Figure 2C). Thermoplastics have the ability to be reshaped when heated near the glass transition temperature $\left(T_{g}\right)$ of the material. Examples of thermoplastics used in hot embossing include polymethylmethacrylate
(PMMA) (Figure 1C), polycarbonate (PC), cyclic olefin copolymer (COC), polystyrene (PS), polyvinylchloride (PVC), and polyethyleneterephthalate glycol (PETG) $(7,33)$.

Typically, masters are made in either silicon or metal. Silicon wafers are processed using micromachining techniques to create a silicon stamp (13-16), while metal stamps are either electroplated against micromachined silicon masters or are electroforms generated using the LIGA process (a German acronym for lithography, electroplating, and molding) (34-36). Once created, the stamp and the selected thermoplastic are placed into a hydraulic press, and then heat and pressure are applied to emboss the plastic against the stamp. Embossing can also be accomplished without heat by using greater amounts of applied pressure (room temperature imprinting) (15).

Embossing is fairly straightforward, as well as fast and inexpensive, provided there is access to the necessary hydraulic press equipment and a patterned stamp. Fabrication of the embossing stamp can be a time-consuming process, and as such, embossing is ideal for situations involving routine microfluidic designs (i.e., not ideal for prototyping a few devices for testing). Specialized vacuum presses are often used in embossing to eliminate air bubbles trapped between the substrate and stamp and provide for precise replications. The replication capability of embossing is limited by the process (e.g., micromachining or LIGA) used for fabrication of the embossing stamp. Most embossed channel systems are one-layer planar structures.

\section{Injection Molding}

Injection molding is a process commonly used in the plastics industry to form a variety of everyday objects. Briefly, prepolymerized pellets of a thermoplastic (e.g., PC, PMMA) are melted and injected under high pressure into the heated mold cavity (Figure 2D). The injected pieces are then cooled below the $\mathrm{T}_{\mathrm{g}}$ of the material and released from the mold (34).

The molten nature of the plastic 
during injection allows for excellent contact with the features of the mold, which results in precise replication. Parameters such as operating temperature and pressure, as well as residence time, greatly affect the degree of contact and thus must be optimized for each production run. Once the operational parameters are optimized, the quality of the replication depends greatly on the quality of the fabricated mold masters, which are produced similarly to masters used for embossing (e.g., micromachined silicon or metal electroforms). For microfluidics, injection molding is more commonly found in industrial applications than in research laboratories due to the complexity of the molding equipment and the fabrication of the masters. In comparison with embossing, injection molding offers higher throughput, in that just a few seconds are required to mold each piece, and the process is easily repeated for large-volume production.

\section{Laser Ablation}

Laser ablation involves the use of a high-powered pulsed laser to remove material from a sheet of thermoplastic. Pulsed excimer UV lasers, capable of pulse rates on the order of $10-10^{4} \mathrm{~Hz}$, are often used in laser ablation as most plastics absorb UV light. ArF excimer lasers $(193 \mathrm{~nm})$ have been used to ablate PS, PC, cellulose acetate, and polyethyleneterephthalate (PET). KrF excimer lasers $(248 \mathrm{~nm})$ have been used with PMMA, PETG, PVC, PC, and polyimide $(37,38)$. A pulse energy of a few hundred $\mathrm{mJ}$ can usually ablate approximately $0.5 \mu \mathrm{m}$ of material. In addition to pulse energy, the depth of the ablated channels is also dependent upon the pulse rate and the absorption characteristics of the substrate. The minimal width of the ablated channel is determined by the focusing optics used to control the beam shape. $\mathrm{CO}_{2}$ lasers, with wavelengths in the infrared region $(10.6 \mu \mathrm{m})$, are also used for microfabrication, typically with PMMA (Figure 1D) or PET (39-41).

A metal mask (lithographic mask) can be used to designate areas to be protected and areas to be exposed to the laser for ablation (Figure 2E). Alternatively, a direct-write process can be used for pattern transfer, which typically involves the use of a programmable high-resolution and highstability translation stage on which the plastic substrate rests. Programmed translation of the stage according to the specified pattern results in the desired channel system. Laser ablation for the fabrication of plastic microfluidic devices is advantageous for prototyping applications, because new microfluidic designs are easily programmed into the system when using the direct- write process. The disadvantage of direct-write laser ablation process is its inherent serial nature, which severely limits its throughput.

\section{ON-CHIP FUNCTIONS}

The functions performed on microfluidic chips are universal. For example, all microfluidic devices, both glass and disposables, must be able to control fluid flow on the microscale. This

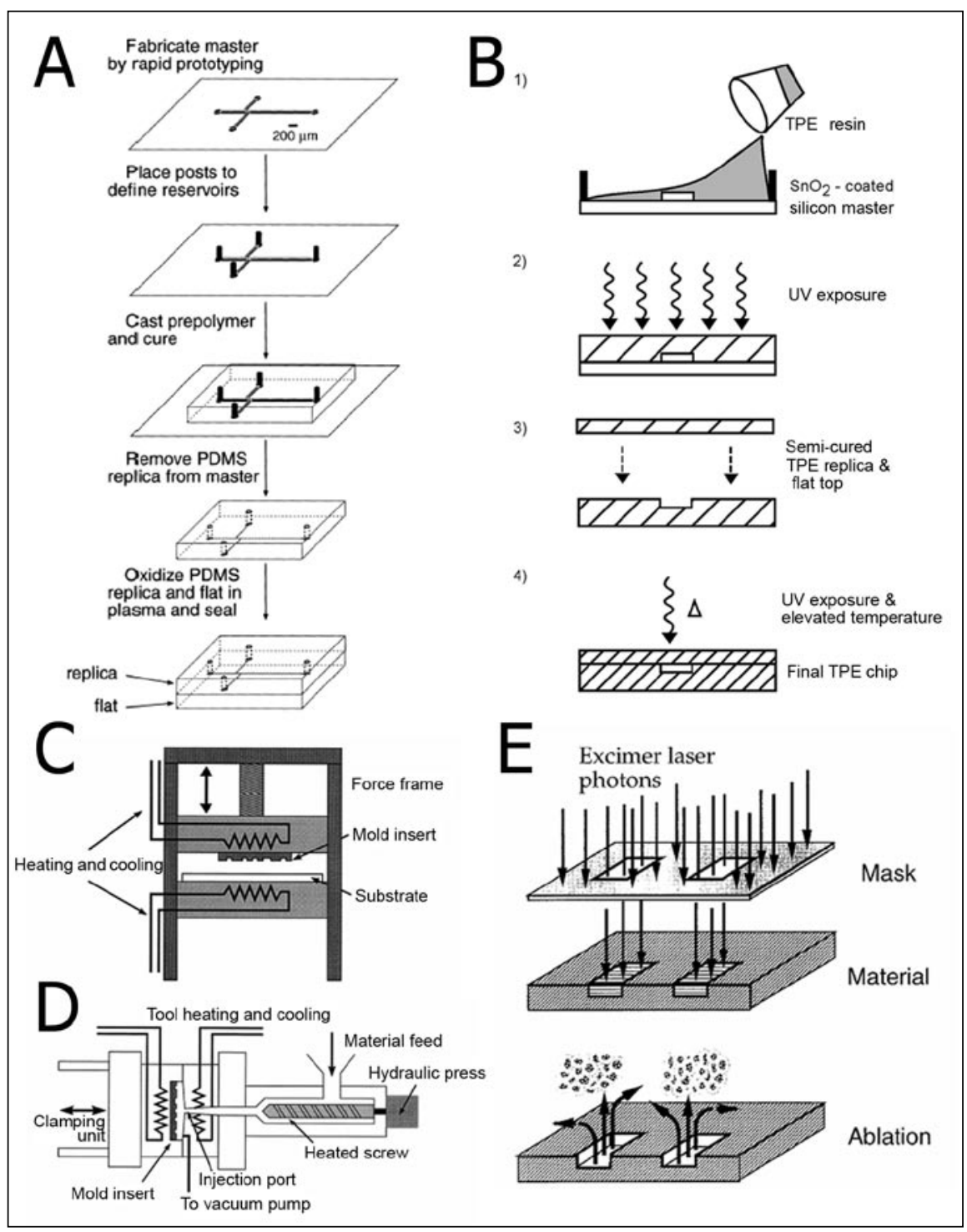

Figure 2. Different fabrication methods for disposable polymer microfluidic devices. Fabrication of (A) polydimethylsiloxane (PDMS) and (B) thermoset polyester (TPE) devices by replica molding, and fabrication of plastic devices using (C) embossing, (D) injection molding, and (E) laser ablation. (A) Reprinted with permission from Reference 20, () 1998, American Chemical Society; (B) reproduced with permission from Reference 31, ( ) 2004, American Chemical Society; (C and D) reprinted from Reference 8 by permission of Wiley; (E) reprinted with permission from Reference 37, () 1997, American Chemical Society. 
section discusses on-chip operations, including fluidic control, analytical separations, and detection methods, which are commonly performed when using microfluidic devices. Included is information on the basic principles at work, most of which derives from original research performed using glass devices, as well as special considerations regarding functions performed using plastic microfluidic systems.

\section{Fluidic Control: Pumps, Valves, and Mixers}

There is a wide range of methods to generate fluid flow in microfluidic devices, including the use of electroosmotic flow, syringe or vacuum pumps, electrokinetic pumps, membrane actuated pumps, centrifugal force, gas bubbles, ferrofluidic plugs, capillary action, electrohydrodynamic pumps, and magnetohydrodynamic pumps $(42,43)$. In general, on-chip pumping is either externally generated by traditional macroscale pumps or is integrated into microchips in the form of microfabricated components or by taking advantage of innate phenomena (e.g., surface tension). The majority of pumps for microfluidics can be described as being governed by electrokinetic principles or by the mechanism of differential pressure. We will also include discussion on the use of valves for controlling and directing fluid flow with electrokinetic and pressure-driven flow, as well as a section regarding laminar flow and strategies to achieve efficient mixing on the microscale. For fluidic control, plastic chips provide capabilities similar to those of glass chips, with the added benefits of easy multilayer fabrication for complex mixing designs. Additionally, the use of elastomers opens up new possibilities for the implementation of pressuredriven flow on-chip.

Electrokinetic control. Electroosmotic flow (EOF) is a phenomenon that occurs in capillary electrophoresis (CE) and is based on the movement of the bulk solution in a capillary or channel caused by the movement of counterions near the surface of the walls under the influence of an applied electric field. Because flow of the bulk solution is driven close to the surface, the resulting flow has a flat velocity profile (Figure $3 \mathrm{~A}$ ), which results in minimal sample dispersion (44). EOF can be produced in channels with diameters in the range of a few hundred nanometers to a couple hundred microns-perfect for application to microfluidic systems. Similarly, to fused silica capillaries used in CE, glass microfluidic devices, as well as several types of plastic microfluidic systems (e.g., PDMS, PMMA, and TPE), can support EOF $(20,31,37,45)$, in which the positive counterions near the negatively charged surfaces move towards the cathode and drag the bulk solution in the same direction.

In general, plastics have been found to have slower EOF rates than glass chips; however, this is not necessarily a disadvantage. At a given applied potential, a slower EOF rate can correspond to longer analysis times, but alternatively it can also correspond to shorter separation lengths required for an analysis, allowing for more efficient packing of microfluidic channels on a given chip. With plastics, the fabrication method can affect the magnitude of EOF. For instance, Locascio et al. showed that laser-ablated polystyrene channels had a faster EOF rate than polystyrene channels fabricated using embossing, presumably through the incorporation of oxygen-containing functional groups during ablation in a residual air environment (45). Oxygen plasma has been used to treat the surfaces of PDMS, TPE, PET, and $\mathrm{COC}$, resulting in changes in the EOF for those types of chips $(20,31,46,47)$. A disadvantage of using plastics is that the stability of the surface can be poor.

The use of EOF for the control of fluid within microfluidic devices is widespread in the literature, stemming from the easy implementation of EOF and the popularity of CE in the analytical community. Fluidic control based on EOF usually involves simple channel design and methods of fabrication, because these systems do not require mechanical valves or other microfabricated parts. The external control, however, can be more complex, because EOF-based fluid valving requires multiple power supplies and timed switching of voltages among these power supplies. An early appli- 
cation of this capability was the use of EOF-driven directional pumping for electrokinetic injection of a sample plug into a $\mathrm{CE}$ separation channel $(11,48,49)$. Another interesting application is the use of EOF in bead and cell sorting, which we will describe in more detail in a later section.

Differential pressure. While EOF is easy to implement and is a popular pumping mechanism for microfluidics, it is not always appropriate. Nonaqueous solutions may not support EOF. For aqueous solutions, the flow rate generated by EOF is typically slow $(<1 \mathrm{~cm} / \mathrm{s})$ and is highly dependent on the surface charge on the channel wall. The applied electric field used to generate EOF also leads to electrophoretic motion-differential motion and consequential separation of charged particles - an intended effect for electrophoresis but undesirable in other situations. In many applications, therefore, the most straightforward method to drive fluid flow is differential pressure.

Unlike macroscale fluidic systems, however, high-pressure differentials are often required to induce flow in microfluidic devices. With pressure-driven flow, also referred to as Poiseuille flow, the volumetric flow rate $(\mathrm{Q})$ for a circular tube is dependent upon the
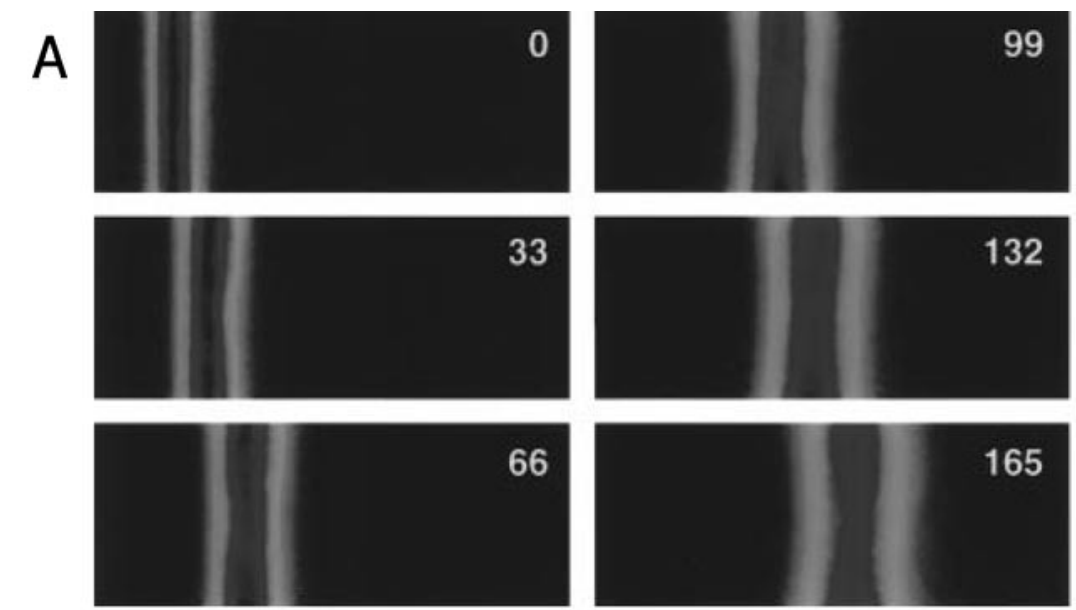

B
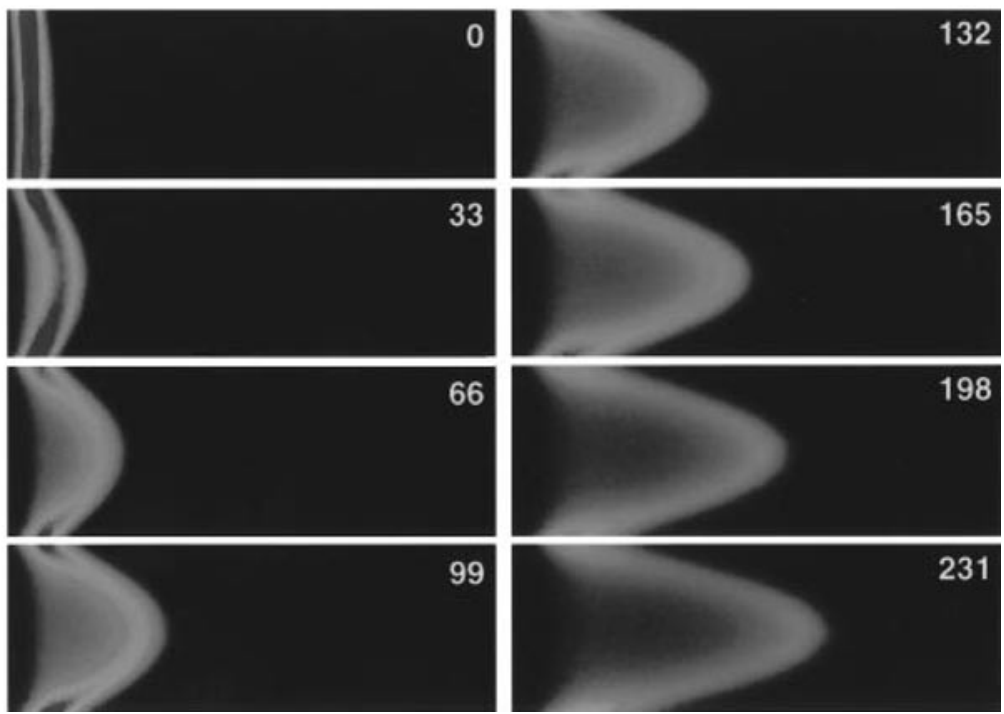

Figure 3. Visualization of flow profiles. Images of (A) electroosmotic flow (EOF) and (B) pressuredriven flow, in 75 and $100 \mu \mathrm{m}$ inner diameter (i.d.) fused silica capillaries, respectively. Image frames are labeled in milliseconds, the elapsed time after the uncaging of a fluorescent dye. Reprinted with permission from Reference 44, (C) 1998, American Chemical Society. applied pressure differential $(\Delta \mathrm{P})$, size of the tube [radius (R) and length (l)], and the viscosity of the solution. Of interest to microchannels is that $\mathrm{Q} \propto$ $(\Delta \mathrm{P})\left(\mathrm{R}^{4}\right)$. Thus, when the tube diameter is decreased by a factor of 100 (e.g., $\mathrm{R}$ decreases from $1 \mathrm{~cm}$ to $100 \mu \mathrm{m}$ ), the $\Delta \mathrm{P}$ applied must be increased by a factor of $10^{8}$ to achieve the same volumetric throughput $(\mathrm{Q})$ as for the macroscale tube. Another aspect of Poiseuille flow is that the velocity of the flow varies with position across the channel, as fluid near the center of the channel moves at a faster rate than fluid near the channel walls, resulting in a parabolic flow profile (Figure 3B) (44). A parabolic flow profile effectively spreads out a given volume of analyte causing sample dispersion, which is disadvantageous for efficient separations.

External pumps (e.g., syringe pumps or vacuum pumps) can easily be used with microfluidic devices to generate pressure-driven flow. Standard plastic tubing is often attached to glass and hard plastic devices through mechanical connectors; simple connections to PDMS devices are accomplished by compression fitting tubing into a bored entry hole. A unique aspect of using pressure-driven flow with elastomeric microfluidic devices is that elastomers respond capacitively to applied pressure, resulting in time delays between the application of differential pressure and the corresponding change in flow rate.

While the use of an external pump (e.g., a syringe pump) offers a convenient way in the research laboratory to drive fluid flow, a major goal of microfluidics is the integration and miniaturization of all necessary components onto a single chip. A common strategy to implement on-chip pressure pumps and valves is the use of deformable membranes. A thin flexible membrane (e.g., PDMS) covers a pump chamber that can be actuated by a number of mechanisms, which ultimately generates repeated movement of the membrane in a manner that drives fluid flow or can maintain the membrane at an open or closed position to gate fluid flow. Some of the mechanisms used with membrane pumps and valves include piezoelectric, electrostatic, and 
thermopneumatic actuation in silicon, glass, and some plastic devices (42), and pneumatic actuation in PDMS devices (26-28). In addition to the capability of achieving integration, membrane pumps are also capable of pumping any type of fluid. Rather than constant flow, membrane-actuated pumping results in pulsatile flow.

Other fluid control principles. Other options for on-chip integration of fluid control include nonmechanical pumps, which typically produce steady flows and are governed by various principles including centrifugal force (50), electrokinetics (51), and capillary action (52). Gyros (Uppsala, Sweden) and Tecan (Maennedorf, Switzerland), commercial producers of compact disc-based microfluidic systems, use injection molding to produce polycarbonate discs imprinted with multiple microchannels (approximately 50-100) and spin the discs to pump fluids by centrifugal force $(53,54)$. Passive gating of fluid in the microchannels is accomplished by capillary burst valves (53), which allow fluid to flow from microchannels into fluid chambers when a rotation frequency threshold is exceeded, thereby overcoming the capillary forces in the microchannel.

Electrokinetic (EK) pumping is another nonmechanical pumping method that is easily integrated on-chip. EK pumps use the pressure differential generated by EOF in hundreds of small diameter $(1-6 \mu \mathrm{m})$ microchannels, which are fabricated in parallel on a chip, for pressure-driven flow in the connecting main electric-field-free microfluidic channel (51). Also, the high fluidic resistance of the narrow EK pump channels prevents fluid from flowing back through the system, acting as an integrated one-way valve. Eksigent Technologies (Livermore, CA, USA) commercially produces microfluidic systems based upon the EK pump principle.

Capillary action was used by Juncker et al. to control fluid within a PDMS microfluidic system without the use of mechanical pumps, EOF, or any other type of external or internal pumping mechanism (52). The researchers used capillary action by controlling surface chemistry and channel geometries to spot protein samples on a solid surface and to perform an immunoassay.

Fluidic mixing. Fluid flow can be described as operating in one of two flow regimes: turbulent or laminar. The Reynolds number (Re) quantitatively characterizes fluid flow; Re above approximately 2300 indicates turbulent flow, while Re below that value indicates laminar flow (for water in a smooth circular channel). $\mathrm{Re}$ is directly proportional to channel diameter, and as such, Re values $<1$ are common in microfluidics, indicating the usual presence of laminar flow in microchannels. A natural consequence of laminar flow in microfluidic systems is that multiple streams can flow sideby-side without turbulent mixing (only diffusional mixing occurs; Figure 4A), which is an advantage in some applications, but problematic for situations requiring rapid mixing of solutions in microchannels.

Diffusional mixing is fast in the nanoscale, but slow in the micro- and macroscale, owing to the nonlinear dependence of diffusion time with distance $\left(x^{2}=2 D t\right)$. For example, fluorescein, a small molecule with $\mathrm{D}$ $=4.25 \times 10^{-10} \mathrm{~m}^{2} / \mathrm{s}$, diffuses $5 \mu \mathrm{m}$ in $<30 \mathrm{~ms}, 50 \mu \mathrm{m}$ in $3 \mathrm{~s}$, and $500 \mu \mathrm{m}$ in approximately $5 \mathrm{~min}(55)$. A variety of strategies have been introduced to achieve fast mixing in microfluidic devices. One strategy focuses on improving interfacial contact of fluid streams for efficient diffusion, such as in the lamination of fluid streams where fluid streams are repeatedly split and recombined $(56,57)$. Another strategy for mixing is through chaotic advection. In this approach, researchers introduce unique channel designs, including bas-relief structures on channel floors (Figure 4B) (58) and 3dimensional serpentine microchannels (Figure 4C) (59) to induce chaotic advection. In general, for lamination or chaotic advection mixing on-chip, in which multidimensional channel designs can be required, disposable devices have an advantage over glass devices due to the ease of multilayer fabrication in plastics.

\section{Analytical Separations}

Besides manipulating fluids inside microfluidic devices, other types of 
on-chip operations include chemical separations that are performed on-chip for analytical purposes. Chromatography and electrophoresis are two major classes of separations commonly implemented on-chip. Each has several variant techniques that have been used in microfluidic devices.

Chromatography. One of the original microfluidic devices was a gas chromatograph (GC) incorporating a $1.5-\mathrm{m}$-long separation column micromachined into a silicon wafer and equipped with an integrated thermal conductivity detector (9). Introduced in 1978, this micro-GC remained the only example of an analytical microfluidic device until the late 1980s, when silicon and glass microfluidic devices were demonstrated for liquid chromatography (LC) and electrophoresis applications $(11,60)$.

From that point on, minimal research was done in the area of micro-GCs, and most microfluidic devices were geared towards liquid applications with a marked emphasis on electrophoresis applications. Throughout the years, LC has seen slow improvements in regards to miniaturization onto chips, in part due to challenges associated with fluidic interconnects and the high pressures required for fluid flow and due to challenges associated with packing stationary phases (61). While some work has been reported with

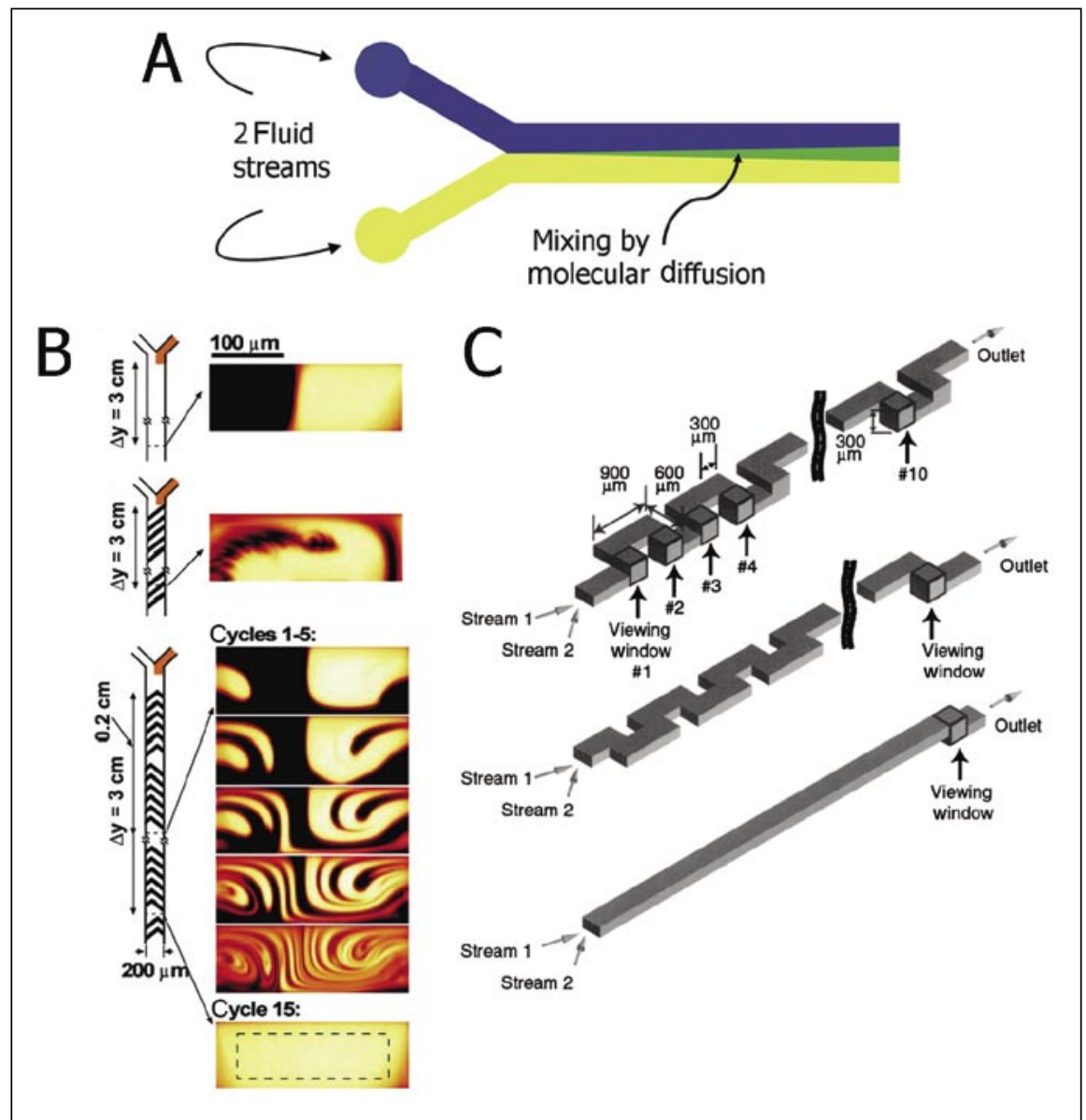

Figure 4. Diffusion and mixing in microfluidic devices. (A) Representation of mixing by diffusion in microchannel. (B) Schematics of a straight channel (top), a channel with raised, straight ridges (middle), and a staggered herringbone mixer (bottom) where mixing by chaotic advection takes place; images to the right of the schematics are the corresponding vertical cross-sectional views of the two fluid streams (a clear fluid stream and a stream that contains fluorescent dyes) that are flowing side by side in the channel. (C) Schematic of a 3-dimensional channel mixer with three orthogonal $90^{\circ}$ turns (top), shown for comparison with a planar channel system with two $90^{\circ}$ turns (middle) and a straight channel with no turns (bottom); mixing by chaotic advection occurs only in the top channel system. (B) Reprinted with permission from Reference 58, (c) 2002, AAAS; (C) reprinted with permission from Reference 59, () 2000, IEEE.

traditional coated silica beads (62), Regnier and coworkers used an interesting approach of micromachining monolithic columns in quartz chips as an alternative to working with packed stationary phases (63), and many more researchers have turned to polymerization and sol-gel chemistries $(61,64)$. However, the issues with integrating pressure-driven fluid flow seem to outweigh packing difficulties. Most of the success with pressure-driven flow for micro-LC devices has been accomplished by industrial companies, including Nanostream (Pasadena, CA, USA) and Eksigent Technologies (64). Nanostream solves interfacing pressure-driven flow to chips by splitting flow from a traditional external pump over 24 on-chip micro-LC columns, while Eksigent Technologies utilizes pressure differentials generated by on-chip electrokinetic pumps. Both companies are currently manufacturing glass chips. The compatibility of chromatography solvents with plastics is of concern for the implementation of disposable microfluidic devices to chromatography applications.

As briefly alluded to, substantial work has been done with incorporating other types of materials [continuous polymer beds (65), porous polymer monoliths $(66,67)$, and sol-gels $(68$ 70)] into microchannels for chromatographic separations. Most researchers investigating integration of alternative packing materials have opted for the use of electric fields for electrokinetic flow instead of attempting to interface pressure-driven flow with packed channels, leading to the current widespread use of on-chip capillary electrochromatography (CEC) (6568,71-74).

Electrophoresis. In contrast to chromatography, CE has become widely used for separations on-chip. Benefits for CE on-chip include the moderately simple implementation of EOF for flow control and easy sample injection of small sample plugs through the use of cross-T, double-T, or other microfluidic designs, as well as the flat flow profile that results from EOF and minimizes sample dispersion (see Electrokinetic Control section above). Due to the extra material surrounding the separation channel, microfluidic chips have a 
greater ability than capillaries to effectively dissipate Joule heat, and therefore, increased voltages can be used on-chip with fewer adverse effects (75).

A significant amount of work in the area of microchip CE focuses on obtaining fast and efficient separations on-chip (12,76,77). Efforts to increase separation efficiencies have included using long separation channels, as well as the use of sieving matrices in the separation channels. In order to provide additional separation length, yet also maintain the compact size of microfluidic chips, channels are packed in limited areas by using serpentine designs. An unwanted effect of packing is the dispersion of analyte bands as a consequence of molecules on the inner radius of a turn having a shorter path than molecules on the outer radius of a turn. Researchers have been able to minimize dispersion due to channel turns by implementing tapered turns or spiral designs (77-80).

The most common CE mode implemented on-chip has been capillary zone electrophoresis (free-solution CE); other modes implemented on-chip include micellar electrokinetic chromatography
(MEKC) (81), isoelectric focusing (IEF) $(82,83)$, and gel electrophoresis. Gel electrophoresis applications have included the use of linear polyacrylamide gels $(75,84,85)$, agarose $(86)$, and other sieving materials $(87,88)$. In addition to the application of the individual CE modes on-chip, researchers have worked to design 2-dimensional systems onchip, for example, incorporating MEKC with free-solution CE (89) or IEF with free-solution CE (90).

Glass microfluidic devices_ranging from soda lime and borosilicate glasses to quartz-are most commonly used for $\mathrm{CE}$ applications. However, in the last few years, disposable microfluidic devices have been applied to microchip CE. Materials for microchip CE have included PDMS (20), COC (16), PMMA (13,34), PET (91), and TPE (31). The material properties relevant to the successful use of CE on polymer chips include sufficient dielectric strength and thermal conductivity, as well as the presence of surface charges for support of EOF. A high dielectric strength enables a material to confine applied potentials to the electrophoretic medium, preventing arcing across the

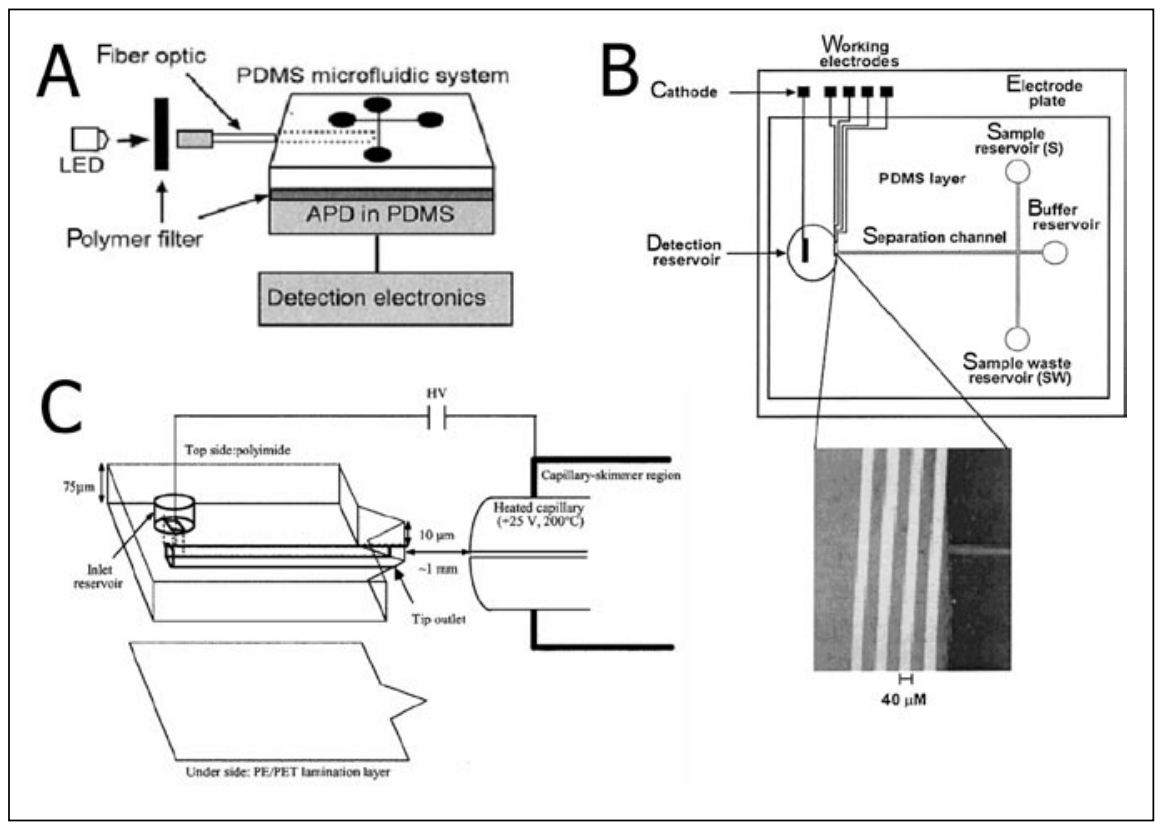

Figure 5. Detection schemes. (A) Schematic of an optical fiber and $\mu$ APD (Avalanche Photodiode) integrated in a polydimethylsiloxane (PDMS) device for on-chip fluorescence detection. (B) Schematic of a microchip capillary electrophoresis (CE) device implementing electrochemical detection and an optical micrograph of working electrodes positioned at the end of a microchannel. (C) Schematic of the positioning of a polymer microchip for electrospray generation and interfacing with a mass spectrometer. (A) Reprinted from Reference 103 by permission of Wiley; (B) reprinted with permission from Reference 118, @ 2000, American Chemical Society; (C) reprinted from Reference 129 by permission of Wiley. 
material itself. Thermal conductivity is important in that Joule heating occurs in a microchannel upon applying a field to drive EOF, and a material with a low thermal conductivity will not be able to adequately dissipate the heat. In general, the thermal conductivities of polymers are not as large as glass, but polymers have been shown to be sufficient for use for electrophoretic applications. The surface properties and EOF rates for a variety of polymers have been investigated, with results showing that EOF varies among different polymers, as well as among different fabrication procedures $(37,38)$. In addition, the adsorption of biomolecules (e.g., proteins) on the surfaces of polymers has been studied in relation to loss of analyte (91) or to the modification of surfaces and the subsequent alteration of EOF rates $(37,45)$. Researchers have also focused on modifying the surface of polymer microchips to obtain better control of EOF (92-95).

\section{Detection}

In conjunction with fluid manipulation and chemical separation, detection of analytes is another important on-chip operation crucial to the total analysis of samples. The main detection schemes implemented onchip include: optical (most common), electrochemical, and mass spectrometric methods (Figure 5) (96-99).

Optical detection. Optical detection has seen extensive use in microfluidics primarily due to ease of implementation and reasonable equipment requirements (96). Fluorescence is the most popular and widely used of the optical detection methods for microfluidics due to its excellent sensitivity, which is often required because of the small sample volumes involved. Off-chip fluorescence detection is typically accomplished through the use of lasers for excitation of fluorescent molecules and charge-coupled device (CCD) cameras or photomultiplier tubes (PMT) for detection of the emitted fluorescent light; fluorescence microscopes are also commonly used. The use of such bulky external light sources and detectors severely constrains the portability of microfluidic devices. Conse- quently, efforts have been focused on miniaturizing the external equipment associated with optical detection (100), as in the microfabrication of lasers (e.g., vertical cavity light emitting diodes) (101) and detectors $(102,103)$, for a true lab-on-a-chip device. Despite the excellent sensitivity of fluorescence detection (single-molecule detection is possible) (104-106), there exists a significant drawback to using fluorescence detection. It is often necessary to fluorescently label samples, as most samples are not inherently fluorescent. Samples can be labeled before (107), during (108), or after (109-111) onchip separations or other forms of sample manipulation. Fluorescent labeling is dependent upon available fluorescent dyes and reaction chemistries, but due to the popularity of fluorescence detection, this area is well developed and there are many options.

Absorbance detection is a straightforward analytical technique often used in conjunction with traditional LC and $\mathrm{CE}$, as most analytes absorb UV light; yet, the use of absorbance detection in microfluidics lags behind fluorescence. The small dimensions of microfluidic channels limit the path length available for absorbance measurements, severely diminishing the sensitivity of absorbance measurements on-chip. Thus, most work in the area of absorbance detection with microfluidics revolves around the search for ways to increase the available path length, thereby increasing sensitivity, or ways for more efficient light collection, thereby minimizing losses $(83,112)$.

In addition to fluorescence and absorbance detection, other optical detection methods used with microfluidics include refractive index detection, Raman spectroscopy, and surface plasmon resonance. Chemiluminescence and electroluminescence are also used with microfluidics. Each of these techniques has its own set of advantages and drawbacks. When considering using optical detection methods with disposable plastic microfluidic devices, special care must be taken in selecting an appropriate material. The chip material must be transparent to light, specifically in wavelength regions equivalent to the sampling wavelength or, for fluorescence, the excitation and emission wavelengths. Most polymers are suitable for use with visible and UV wavelengths (e.g., PDMS, PMMA, COC). Also, in the case of fluorescence detection, the material should exhibit no or minimal autofluorescence, which leads to a high background signal and a corresponding reduction in sensitivity. Hawkins and Yager determined that PET films, a common substrate for microfluidics, exhibits more autofluorescence than COC, PMMA, PC, polypropylene, or polystyrene (113).

Electrochemical detection. Microfluidic devices that use electrochemical detection (ECD) $(97,98)$ have the potential to be very compact and fully integrated, because the analyte signal is detected through the use of electrodes, which are by nature small and compact. Analyte molecules that undergo either oxidation or reduction reactions can be easily detected using ECD; nonelectroactive compounds can be detected with the use of derivatization agents (similar to dye tagging in fluorescence). In amperometric detection, which is more commonly used in conjunction with microfluidics than potentiometry, the potential at the working electrode is set at a fixed value to drive the redox reaction, and the output current is monitored as a function of time, thereby providing information about the traversing chemical species in the microchannel. Conductivity detection has also been implemented with microfluidic devices $(35,114-116)$.

Coupling amperometric detection to microfluidic devices has been typically accomplished by using metal deposition techniques or thick-film technologies (e.g., electroless deposition, evaporation, sputtering, screen printing) to pattern electrodes within the devices during the microfabrication process. The most common electrode materials are carbon, platinum, and gold. A significant portion of research in the area of on-chip amperometric detection focuses on the electrical isolation of the detection electrodes from the high voltages used for CE separation, as the potentials used for amperometric detection are orders of magnitude smaller than typical separation voltages. Many researchers have opted to locate the working electrode just outside of the microchannel (117-119) or physi- 
cally at the microchannel exit (120). Some reports point to the possibility of using in-channel electrodes under adequate electrical isolation $(121,122)$. Early demonstrations of amperometric detection on-chip were presented in glass and quartz chips by the Ewing and Mathies groups $(117,123)$, but plastic chips have recently been introduced, including the use of PDMS (118) and PET (46). For composite PDMS/glass devices, the electrodes have been located on the glass substrate, as electrodes patterned on PDMS can crack if the elastomer is flexed.

Mass spectrometry. Integrating mass spectrometry (MS) with microfluidics has become a significant effort in recent years as the power of MS for analyte identification at low concentrations greatly complements the power of microfluidics for small volume sample handling and efficient separations (99). The typical flow rates used in microfluidics ( $\mathrm{nL}$ to $\mu \mathrm{L} / \mathrm{min}$ ) are well suited for electrospray ionization mass spectrometry (ESI-MS). The integration of the ionization process onto microfluidic devices, however, is challenging owing to the planar nature of microfabricated systems. As such, much work in the field deals with ways to create suitable tips for electrospray on-chip.

One method for interfacing electrospray with microfluidic devices involves gluing or bonding electrospray emitters, such as fused silica capillaries or nanospray needles, to the end of the chip at the outlet of the microchannel (99). A variation of this method is attaching an external liquid junction to the chip (124). For coupling CE to MS, there are two main issues to deal with: (i) the low compatibility of the CE buffer with ESI and (ii) the necessity to decouple the two (electrophoresis and electrospray) electric fields. The use of an external liquid junction handles both of these issues fairly well by providing a solvent make-up and sheath flow to the effluent, as well as isolating the electrospray electric field from the onchip separation voltages.

Another method for integrating MS with microfluidics is to integrate electrospray tips during the chip fabrication process. Original efforts for integrated on-chip electrospray were achieved using glass chips, with spray emitted from the microchannel outlet at the edge of the chip $(125,126)$, while recent advances have involved fabrication of on-chip electrospray in a variety of materials, such as PET, polyimide, PDMS, parylene, PC, and PMMA (127). Fabrication of the electrospray tip can be as simple as cutting or ablating the tip to the desired shape, as demonstrated by Girault and coworkers, for the integration of ESIMS with photoablated/plasma-etched PET/polyimide microfluidic devices $(128,129)$. The use of fabrication for on-chip integration has the advantage 
that the electrodes used to generate the required electric field can be patterned during the fabrication process, and sheath flow channels can be integrated with electrophoresis channels for chipbased CE-MS (130).

\section{MICROFLUIDIC DEVICES FOR BIOANALYTICAL APPLICATIONS}

\section{Nucleic Acid Analysis}

Currently, one of the leading applications of microfluidic devices is nucleic acid analysis. Due to the large body of work for chip-based DNA analysis systems, we will not be able to give a comprehensive review of this area, and we refer readers to reviews by Landers (88) and Verpoorte (131) for in-depth summaries of the research to date. Early demonstrations of the applicability of CE-based DNA analysis onchip included separations of oligonucleotides (10-25 bases) (75) and sizing of longer DNA fragments ( $\Phi \times 174$ HaeIII digest sizing ladder, 70-1000 bp) (87). More recently, microfluidic devices are being applied to genotyping experiments for screening of hereditary diseases or for forensic analyses $(88,131)$. A significant advantage for using microfluidic devices is the decreased analysis time. DNA sizing on-chip is typically 10 times faster than using CE capillaries and 100 times faster than the traditional slab-gel format (132). DNA separations on-chip are typically accomplished with glass devices using a sieving matrix (e.g., linear polyacrylamide) in the microchannels and laser-induced fluorescence (LIF) detection. High-resolution separations for DNA sequencing were the next logical extension for on-chip DNA analysis, and the Mathies group first demonstrated on-chip DNA sequencing in 1995 (84). Since then, glass microfluidic devices with 96 and 384 microchannels have been introduced (133-135), as well as the use of four-color detection schemes (136).

In addition to electrophoretic separations of DNA on-chip, another significant application for microfluidic devices is DNA amplification. Implementation of PCR on-chip has been accomplished using several different approaches (137). However, the primary advantage for PCR onchip is the decreased thermal cycling times, which translate to shorter overall reaction times. Approaches have varied from simple silicon microreaction chambers (138) to a continuous flow PCR system (139). Coupling PCR with $\mathrm{CE}$ separations on-chip has been of

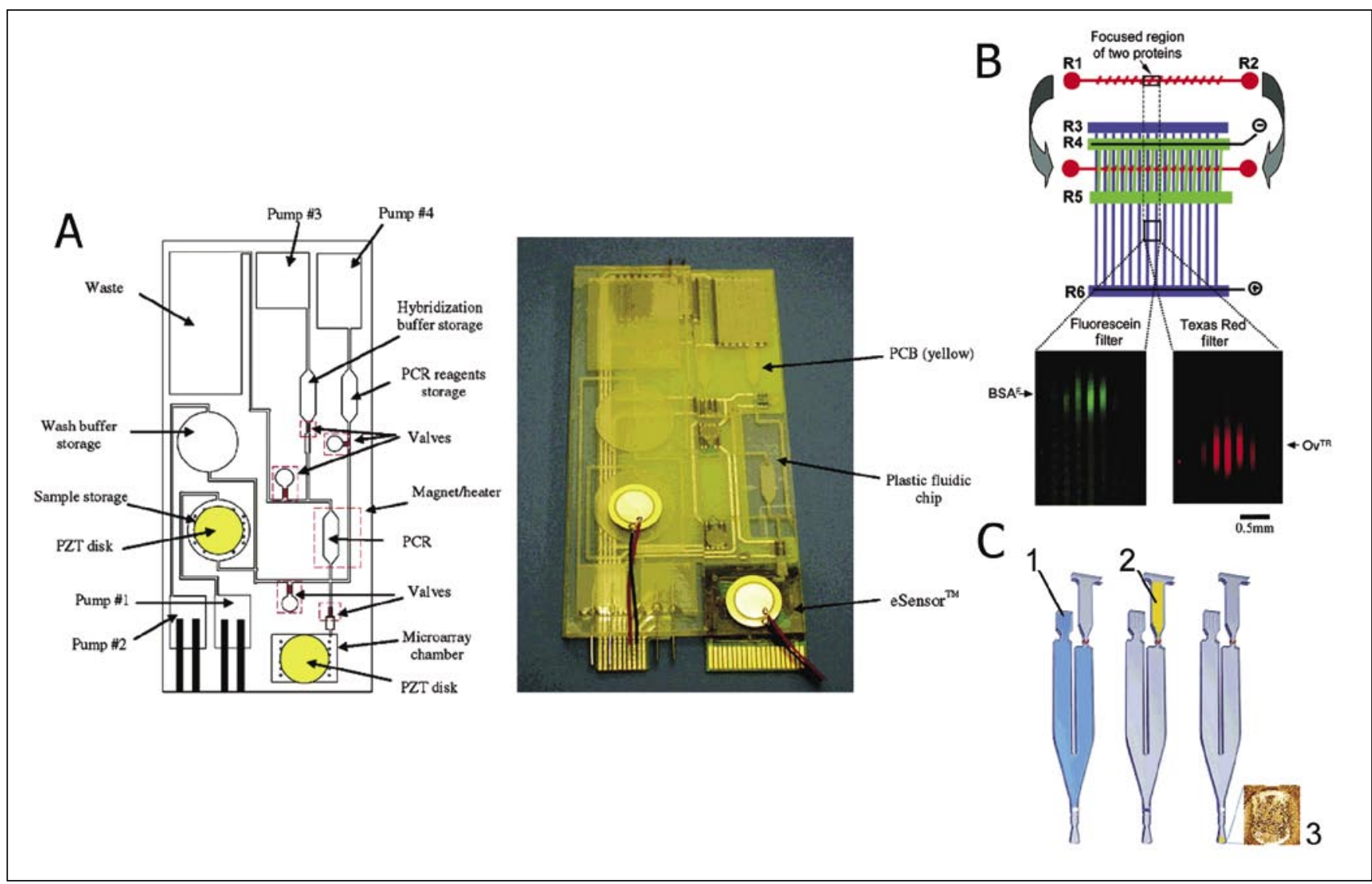

Figure 6. On-chip DNA and protein analysis. (A) Schematic and photograph of an integrated device for sample preparation, PCR, and DNA microarray detection. (B) Schematic of a 2- dimensional capillary electrophoresis (CE) microfluidic device, in which the membrane layer containing the isoelectric focusing (IEF) microchannel is positioned perpendicularly to the microchannels used for the 2-dimensional separation. (C) Schematic of a microfluidic structure for sample preparation and matrix-assisted laser desorption ionization mass spectrometry (MALDI MS); locations for sample application (1), washing and elution (2), and co-crystallization (3). (A) Reprinted with permission from Reference 144, (C) 2004, American Chemical Society; (B) reprinted with permission from Reference 86, ( ) 2002, American Chemical Society; (C) reprinted with permission from Reference 54, ( 2004, American Chemical Society. 
interest (140), as well as the integration of sample preparation or cell lysis with PCR and CE on-chip (141-143).

Most of the microfluidic devices used to date for DNA sizing, genotyping, sequencing, and PCR amplification have been fabricated in glass. However, polymeric materials recently have been used for chip-based nucleic acid analysis (144-149). For example, Liu et al. (144) reported a fully integrated microfluidic device capable of sample preparation, PCR, and subsequent electrochemical detection through DNA hybridization to an incorporated microarray (Figure $6 \mathrm{~A})$. Whole blood samples $(1 \mathrm{~mL})$ are applied to the chip, and target cells are captured by magnetic beads on-chip and then lysed for analysis. The system is unique in that all fluid manipulation components (mixers, valves, and pumps) are integrated on-chip.

\section{Protein Analysis}

The additional chemical complexity of proteins has made their analysis onchip more difficult than that of DNA. Protein analysis on-chip encompasses a wide range of applications and technical developments, including immunoassays, enzyme assays, integration with mass spectrometry, methods for sample preparation, as well as considerable research on separations of amino acids, peptides, and proteins-informative reviews have been presented by Lion et al. (99) and Verpoorte (131). Separations on-chip began with demonstrations of 1-dimensional separationssodium dodecyl sulfate (SDS) gel-based molecular sizing $(85,150)$, IEF $(82,83)$, CEC $(65,67,74)$, MEKC (77)—and, in the context of proteomics, have more recently focused on integrating orthogonal separations to create 2dimensional separation systems with correspondingly increased peak capacities $(89,90,151)$.

In an effort to miniaturize traditional 2-dimensional slab-gel electrophoresis of proteins onto microchip, Herr et al. (90) coupled IEF with CE in a PMMA device. IEF was accomplished in the presence of EOF, in which proteins were focused while in motion towards an intersection with the second dimension-free solution CE channel, and specific focused bands could be selected for further analysis by halting the IEF step and injecting the bands into the $\mathrm{CE}$ channel for further separation. Other approaches have involved the use of many parallel microchannels orthogonal to a main 1-dimensional separation channel $(86,152-154)$. The approach of Chen et al. (Figure 6B) (86) for 2-dimensional protein analysis, for example, was to use a multilayer PDMS device for gel-based IEF coupled to SDS gel electrophoresis. Agarose was used as the gel medium. After focusing proteins in the IEF channel, the layers of the PDMS device were peeled apart, and the layer containing the IEF gel was coupled to the 100 parallel SDS gel electrophoresis microchannels for the second-dimension separation.

Besides 2-dimensional separations, the application of MS to protein analysis is another interesting application for microfluidic chips. As discussed in the Mass Spectrometry Detection section, a significant portion of research has focused on coupling microchips with ESI. Research by Gustafsson et al. (54), however, presented the use of disposable microchips for the preparation of protein samples for matrix-assisted laser desorption ionization (MALDI) MS (Figure 6C). The microfluidic device included 96 microchannels that were injection molded in PC in a compactdisc format and used centrifugal force to direct fluid flow. Samples of protein digest were applied to the microchannel structures, driven into an integrated reverse-phase column for concentration and desalting, and then eluted from the column with solvent and allowed to crystallize with matrix at the outlet of the microchannels - ready for subsequent MALDI MS analysis directly from the microchip.

\section{Cellular Studies}

While the integration of cell lysis with separations on-chip (142) is a logical extension of microfluidics for cellular analysis, there are a variety of other applications pertaining to studies of biological cells on-chip $(19,155)$. We have selected for discussion four examples (see Figure 7)-a microfabricated fluorescence-activated cell 


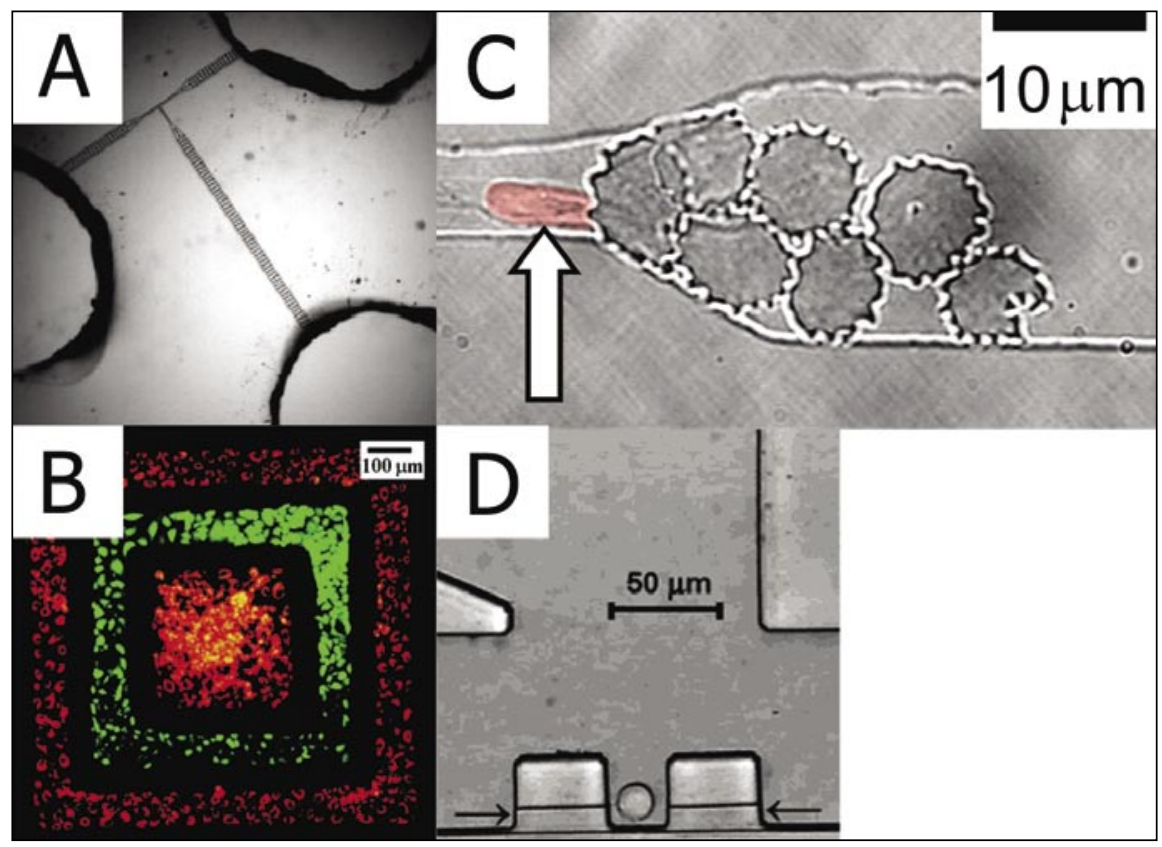

Figure 7. On-chip cellular applications. (A) Optical micrograph of a microfabricated fluorescenceactivated cell sorter $(\mu \mathrm{FACS})$ device. (B) Fluorescence micrograph of two cell types [fluorescently labeled human bladder cancer cells (ECV304 cell line) and bovine adrenal capillary endothelial cells] deposited on a tissue culture dish in a concentric square pattern using multilayer fluidic channels fabricated in polydimethylsiloxane (PDMS). (C) Optical micrograph of a normal red blood cell as it exits a blockage formed by malaria-infected erythrocytes in a PDMS microchannel. (D) Optical micrograph of a single Jurkat T cell captured in a cell dock. (A) Reprinted from Reference 156, (C) 1999, Nature Publishing Group; (B) reprinted from Reference 157, (C) 2000, National Academy of Sciences, USA; (C) reprinted from Reference 158, ( ) 2003, National Academy of Sciences, USA; (D) reprinted in part with permission from Reference 159, (C) 2003, American Chemical Society.

sorter ( $\mu$ FACS) (156), the patterned deposition of cells using complex microchannels (157), a microfluidic device for biophysical modeling of cells (158), and a single-cell capture and analysis system (159) - that are illustrative of the diverse cellular applications possible with disposable polymer-based microfluidic devices.

Flow cytometry is used for a variety of clinical and basic research applications, including disease diagnosis, cell screening, and antibody-labeled bead assays, and chip-based flow cytometry has the potential to be successfully employed in these same applications with increased sensitivity in an inexpensive and disposable format. Early microfabricated flow cytometry devices were based on hydrodynamic focusing (160), electrokinetic focusing (161), as well as a T-channel design (Figure 7A) that used EOF to drive and direct fluid flow for cell sorting (156). Fu et al. later took advantage of the capability to integrate membrane- actuated valves and pumps within polymeric microfluidic devices, reporting the fabrication of a multilayer PDMS device for $\mu$ FACS that was operated by pressure-driven flow (162).

The capability to deposit multiple types of cells on surfaces with precise control over their location provides significant benefits to fundamental studies in cell biology, as well as applied research on cell-based biosensors and tissue engineering. Microfluidics can offer this required spatial control for deposition of biological molecules, materials, and cells $(19,25,163,164)$. For example, Figure 7B shows the use of complex 3-dimensional microfluidic systems to deposit two cell types simultaneously (157). Here cell suspensions were added to the PDMS microchannels and allowed to attach to the surface, followed by a 24-h culture period. Upon removal of the 3-dimensional PDMS stamp, which was sealed conformally to the surface, the cells were allowed to grow and spread together for cell-cell interaction studies and to gain insight into tumor angiogenesis. The patterning of many different cell types in close proximity may also be used to mimic the complex environment of cells in tissues.

Emulating the in vivo environment of blood capillaries is useful for understanding the biomechanics of suspension cells as they flow through such capillaries (165-167). One specific example is malaria-infected erythrocytes that become increasingly inelastic with progression in the stages of infection, causing capillary blockage and ultimately organ failure. With the aid of elastomeric PDMS microchannels, Shelby et al. recently presented a microfluidic in vitro model of capillary obstruction by malariainfected red blood cells (158). Figure 7C shows the tortuous path taken by a normal erythrocyte as it squeezes through a blockage caused by malariainfected erythrocytes at the entrance to a 6- $\mu \mathrm{m}$ channel constriction.

In contrast to the $\mu$ FACS device described above, which actively sorted a population of cells, Wheeler et al. presented a microfluidic device designed to allow the passive capture of a single cell from a bulk solution (159). The device was made from PDMS and incorporated membrane-actuated valve and pumps to direct fluid flow. Figure 7D shows the concept of this device, in which a single cell can be selected and captured at a docking location by manipulation of fluid flow. Additional side inlet channels deliver reagents directly to the docked cell for cell viability assays and measurements of calcium fluxes at the level of single cells.

\section{CONCLUSIONS}

Following early proof-of-principle work in microfluidics with silicon and glass devices, polymer-based microchips have emerged in recent years as inexpensive and disposable alternatives to micromachined devices. With microfabrication methods that range from molding techniques to laser ablation, the fabrication of polymer microchips can be quite fast and versatile, 
applicable to both prototyping and high-throughput production. Polymer devices have also proven to be suitable for an assortment of on-chip functions and applications.

While glass devices will continue to be used for applications requiring harsh conditions or reusability, disposable microchips should offer additional opportunities for integration of components and operations on-chip. Plastics provide a variety of approaches and an ease of fabrication that will enable the design and creation of integrated systems in an efficient and economical fashion. In the future, we expect to see additional examples of methods of detection, sample preparation, separations, and fluid manipulation incorporated onto microchips, with further emphasis on the production of self-contained and truly integrated labon-a-chip systems.

\section{ACKNOWLEDGMENTS}

We thank R. Lorenz for careful reading of the manuscript, as well as the National Institutes of Health (NIH), the National Science Foundation (NSF), the Keck Foundation, and the American Chemical Society Petroleum Research Fund (ACS PRF) for financial support.

\section{COMPETING INTERESTS STATEMENT}

The authors declare no competing interests.

\section{REFERENCES}

1.Reyes, D.R., D. Iossifidis, P.A. Auroux, and A. Manz. 2002. Micro total analysis systems. 1. Introduction, theory, and technology. Anal. Chem. 74:2623-2636.

2.Auroux, P.A., D. Iossifidis, D.R. Reyes, and A. Manz. 2002. Micro total analysis systems. 2. Analytical standard operations and applications. Anal. Chem. 74:2637-2652.

3.Vilkner, T., D. Janasek, and A. Manz. 2004. Micro total analysis systems. Recent developments. Anal. Chem. 76:3373-3386.

4.Schaller, T., L. Bohn, J. Mayer, and K. Schubert. 1999. Microstructure grooves with a width of less than $50[\mathrm{mu}] \mathrm{m}$ cut with ground hard metal micro end mills. Precis. Eng. 23:229-235.

5.Beebe, D.J., J.S. Moore, Q. Yu, R.H. Liu, M.L. Kraft, B.H. Jo, and C. Devadoss. 2000. Microfluidic tectonics: a comprehensive construction platform for microfluidic systems. Proc. Natl. Acad. Sci. USA 97:13488-13493.

6.Rossier, J.S., C. Vollet, A. Carnal, G. Lagger, V. Gobry, H.H. Girault, P. Michel, and F. Reymond. 2002. Plasma etched polymer microelectrochemical systems. Lab Chip 2:145-150.

7.Becker, H. and L.E. Locascio. 2002. Polymer microfluidic devices. Talanta 56:267-287.

8.Becker, H. and C. Gartner. 2000. Polymer microfabrication methods for microfluidic analytical applications. Electrophoresis 21:12-26.

9.Terry, S.C., J.H. Jerman, and J.B. Angell. 1979. A gas chromatographic air analyzer fabricated on a silicon wafer. IEEE Trans. Electron Devices ED-26:1880-1886.

10.Manz, A., D.J. Harrison, E.M.J. Verpoorte, J.C. Fettinger, A. Paulus, H. Ludi, and H.M. Widmer. 1992. Planar chips technology for miniaturization and integration of separation techniques into monitoring systems. J. Chromatogr. 593:253-258.

11.Harrison, D.J., A. Manz, Z. Fan, H. Luedi, and H.M. Widmer. 1992. Capillary electrophoresis and sample injection systems integrated on a planar glass chip. Anal. Chem. 64:1926-1932.

12.Jacobson, S.C., R. Hergenroder, L.B. Koutny, and J.M. Ramsey. 1994. High-speed separations on a microchip. Anal. Chem. 66:1114-1118.

13.Martynova, L., L.E. Locascio, M. Gaitan, G.W. Kramer, R.G. Christensen, and W.A. MacCrehan. 1997. Fabrication of plastic microfluid channels by imprinting methods. Anal. Chem. 69:4783-4789.

14.Becker, H. and U. Heim. 1999. Polymer hot embossing with silicon master structures. Sens. Mater. 11:297-304.

15.Xu, J.D., L. Locascio, M. Gaitan, and C.S. Lee. 2000. Room-temperature imprinting method for plastic microchannel fabrication. Anal. Chem. 72:1930-1933.

16.Kameoka, J., H.G. Craighead, H. Zhang, and J. Henion. 2001. A polymeric microfluidic chip for CE/MS determination of small molecules. Anal. Chem. 73:1935-1941.

17.Rodriguez, I., P. Spicar-Mihalic, C.L. Kuyper, G.S. Fiorini, and D.T. Chiu. 2003. Rapid prototyping of glass microchannels. Anal. Chim. Acta 496:205-215.

18.Lorenz, R.M., C.L. Kuyper, P.B. Allen, L.P. Lee, and D.T. Chiu. 2004. Direct laser writing on electrolessly deposited thin metal films for applications in micro- and nanofluidics. Langmuir 20:1833-1837.

19.Whitesides, G.M., E. Ostuni, S. Takayama, X.Y. Jiang, and D.E. Ingber. 2001. Soft lithography in biology and biochemistry. Ann. Rev. Biomed. Eng. 3:335-373.

20.Duffy, D.C., J.C. McDonald, O.J.A. Schueller, and G.M. Whitesides. 1998. Rapid prototyping of microfluidic systems in poly(dimethylsiloxane). Anal. Chem. 70:4974-4984.

21.McDonald, J.C., D.C. Duffy, J.R. Anderson, D.T. Chiu, H.K. Wu, O.J.A. Schueller, and G.M. Whitesides. 2000. Fabrication of microfluidic systems in poly(dimethylsiloxane). Electrophoresis 21:27-40.

22.McDonald, J.C. and G.M. Whitesides. 2002. Poly(dimethylsiloxane) as a material for fabricating microfluidic devices. Acc. Chem. Res. 35:491-499.

23.Anderson, J.R., D.T. Chiu, R.J. Jackman, O. Cherniavskaya, J.C. McDonald, H. Wu, S.H. 
Whitesides, and G.M. Whitesides. 2000. Fabrication of topologically complex three-dimensional microfluidic systems in PDMS by rapid prototyping. Anal. Chem. 72:3158-3164.

24.Jo, B.H., L.M. Van Lerberghe, K.M. Motsegood, and D.J. Beebe. 2000. Three-dimensional micro-channel fabrication in polydimethylsiloxane (PDMS) elastomer. J. Microelectromech. Sys. 9:76-81.

25.Folch, A. and M. Toner. 2000. Microengineering of cellular interactions. Ann. Rev. Biomed. Eng. 2:227-256

26.Unger, M.A., H.P. Chou, T. Thorsen, A. Scherer, and S.R. Quake. 2000. Monolithic microfabricated valves and pumps by multilayer soft lithography. Science 288:113-116.

27.Ismagilov, R.F., D. Rosmarin, P.J.A. Kenis, D.T. Chiu, W. Zhang, H.A. Stone, and G.M. Whitesides. 2001. Pressure-driven laminar flow in tangential microchannels: an elastomeric microfluidic switch. Anal. Chem. 73:4682-4687.

28.Jeon, N.L., D.T. Chiu, C.J. Wargo, H.K. Wu, I.S. Choi, J.R. Anderson, and G.M. Whitesides. 2002. Design and fabrication of integrated passive valves and pumps for flexible polymer 3-dimensional microfluidic systems. Biomed. Microdevices 4:117-121.

29.Lee, J.N., C. Park, and G.M. Whitesides. 2003. Solvent compatibility of poly(dimethylsiloxane)based microfluidic devices. Anal. Chem. 75:65446554 .

30.Rolland, J.P., R.M. Van Dam, D.A. Schorzman, S.R. Quake, and J.M. DeSimone. 2004. Solvent-resistant photocurable "liquid Teflon" for microfluidic device fabrication. J. Amer. Chem. Soc. 126:2322-2323.

31.Fiorini, G.S., R.M. Lorenz, J.S. Kuo, and D.T. Chiu. 2004. Rapid prototyping of thermoset polyester microfluidic devices. Anal. Chem. 76:4697-4704.

32.Fiorini, G.S., G.D.M. Jeffries, D.S.W. Lim, C.L. Kuyper, and D.T. Chiu. 2003. Fabrication of thermoset polyester microfluidic devices and embossing masters using rapid prototyped polydimethylsiloxane molds. Lab Chip 3:158163.

33.Becker, H. and U. Heim. 2000. Hot embossing as a method for the fabrication of polymer high aspect ratio structures. Sens. Actuators A 83:130135

34.McCormick, R.M., R.J. Nelson, M.G. AlonsoAmigo, J. Benvegnu, and H.H. Hooper. 1997. Microchannel electrophoretic separations of DNA in injection-molded plastic substrates. Anal. Chem. 69:2626-2630.

35.Galloway, M., W. Stryjewski, A. Henry, S.M. Ford, S. Llopis, R.L. McCarley, and S.A. Soper. 2002. Contact conductivity detection in poly(methyl methacylate)-based microfluidic devices for analysis of mono- and polyanionic molecules. Anal. Chem. 74:2407-2415.

36.Qi, S.Z., X.Z. Liu, S. Ford, J. Barrows, G. Thomas, K. Kelly, A. McCandless, K. Lian, et al. 2002. Microfluidic devices fabricated in poly(methyl methacrylate) using hot-embossing with integrated sampling capillary and fiber optics for fluorescence detection. Lab Chip 2:88-95.

37.Roberts, M.A., J.S. Rossier, P. Bercier, and H. Girault. 1997. UV laser machined polymer substrates for the development of microdiagnostic systems. Anal. Chem. 69:2035-2042.

38.Pugmire, D.L., E.A. Waddell, R. Haasch, M.J.
Tarlov, and E. Locascio. 2002. Surface characterization of laser-ablated polymers used for microfluidics. Anal. Chem. 74:871-878.

39.Klank, H., J.P. Kutter, and O. Geschke. 2002. $\mathrm{CO}_{2}$-laser micromachining and back-end processing for rapid production of PMMA-based microfluidic systems. Lab Chip 2:242-246.

40.Jensen, M.F., M. Noerholm, L.H. Christensen, and O. Geschke. 2003. Microstructure fabrication with $\mathrm{CO}_{2}$ laser system: characterization and fabrication of cavities produced by raster scanning of the laser beam. Lab Chip 3:302-307.

41.Snakenborg, D., H. Klank, and J.P. Kutter. 2004. Microstructure fabrication with a $\mathrm{CO}_{2}$ laser system. J. Micromech. Microeng. 14:182-189.

42.Laser, D.J. and J.G. Santiago. 2004. A review of micropumps. J. Micromech. Microeng. 14: R35-R64.

43.Beebe, D.J., G.A. Mensing, and G.M. Walker. 2002. Physics and applications of microfluidics in biology. Ann. Rev. Biomed. Eng. 4:261-286.

44.Paul, P.H., M.G. Garguilo, and D.J. Rakestraw. 1998. Imaging of pressure- and electrokinetically driven flows through open capillaries. Anal. Chem. 70:2459-2467.

45.Locascio, L.E., C.E. Perso, and C.S. Lee. 1999. Measurement of electroosmotic flow in plastic imprinted microfluid devices and the effect of protein adsorption on flow rate. J. Chromatog. A 857:275-284

46.Rossier, J., F. Reymond, and P.E. Michel. 2002. Polymer microfluidic chips for electrochemical and biochemical analyses. Electrophoresis 23:858-867.

47.Gaudioso, J. and H.G. Craighead. 2002. Characterizing electroosmotic flow in microfluidic devices. J. Chromatogr. A 971:249-253.

48.Seiler, K., D.J. Harrison, and A. Manz. 1993. Planar glass chips for capillary electrophoresis: repetitive sample injection, quantitation, and separation efficiency. Anal. Chem. 65:1481-1488.

49.Jacobson, S.C., R. Hergenroder, L.B. Koutny, R.J. Warmack, and J.M. Ramsey. 1994. Effects of injection schemes and column geometry on the performance of microchip electrophoresis devices. Anal. Chem. 66:1107-1113.

50.Zoval, J.V. and M.J. Madou. 2004. Centrifugebased fluidic platforms. Proc. IEEE 92:140-153.

51.Lazar, I.M. and B.L. Karger. 2002. Multiple open-channel electroosmotic pumping system for microfluidic sample handling. Anal. Chem. 74:6259-6268.

52.Juncker, D., H. Schmid, U. Drechsler, H. Wolf, M. Wolf, B. Michel, N. de Rooij, and E. Delamarche. 2002. Autonomous microfluidic capillary system. Anal. Chem. 74:6139-6144.

53.Duffy, D.C., H.L. Gillis, J. Lin, N.F. Sheppard, Jr., and G.J. Kellogg. 1999. Microfabricated centrifugal microfluidic systems: characterization and multiple enzymatic assays. Anal. Chem. 71:4669-4678.

54.Gustafsson, M., D. Hirschberg, C. Palmberg, H. Jornvall, and T. Bergman. 2004. Integrated sample preparation and MALDI mass spectrometry on a microfluidic compact disk. Anal. Chem. 76:345-350.

55.Culbertson, C.T., S.C. Jacobson, and J. Michael Ramsey. 2002. Diffusion coefficient measurements in microfluidic devices. Talanta 56:365-373.

56.Branebjerg, J., P. Gravesen, J.P. Kog, and C.R. Nielsen. 1996. Fast mixing by lamination. Proc.
IEEE MEMS Workshop, San Diego, p. 441-446.

57.Schonfeld, F., V. Hessel, and C. Hofmann. 2004. An optimised split-and-recombine micromixer with uniform 'chaotic' mixing. Lab Chip 4:65-69.

58.Stroock, A.D., S.K.W. Dertinger, A. Ajdari, I. Mezit, H.A. Stone, and G.M. Whitesides. 2002. Chaotic mixer for microchannels. Science 295:647-651.

59.Liu, R.H., M.A. Stremler, K.V. Sharp, M.G. Olsen, J.G. Santiago, R.J. Adrian, H. Aref, and D.J. Beebe. 2000. Passive mixing in a three-dimensional serpentine microchannel. J. Microelectromech. Sys. 9:190-197.

60.Manz, A., Y. Miyahara, J. Miura, Y. Watanabe, H. Miyagi, and K. Sato. 1990. Design of an open-tubular column liquid chromatograph using silicon chip technology. Sens. Actuators B B1:249-255.

61.de Mello, A. 2002. On-chip chromatography: the last twenty years. Lab Chip 2:48N-54N.

62. Ocvirk, G., E. Verpoorte, A. Manz, M. Grasserbauer, and H.M. Widmer. 1995. High performance liquid chromatography partially integrated onto a silicon chip. Anal. Methods Instrum. 2:7482.

63.He, B., N. Tait, and F. Regnier. 1998. Fabrication of nanocolumns for liquid chromatography. Anal. Chem. 70:3790-3797.

64.Harris, C.M. 2003. Shrinking the LC landscape Anal. Chem. 75:64A-69A.

65.Ericson, C., J. Holm, T. Ericson, and S. Hjerten. 2000. Electroosmosis- and pressuredriven chromatography in chips using continuous beds. Anal. Chem. 72:81-87.

66.Yu, C., F. Svec, and J.M.J. Frechet. 2000. Towards stationary phases for chromatography on a microchip: molded porous polymer monoliths prepared in capillaries by photoinitiated in situ polymerization as separation media for electrochromatography. Electrophoresis 21:120-127.

67.Throckmorton, D.J., T.J. Shepodd, and A.K. Singh. 2002. Electrochromatography in microchips: reversed-phase separation of peptides and amino acids using photopatterned rigid polymer monoliths. Anal. Chem. 74:784-789.

68.Constantin, S., R. Freitag, D. Solignac, A Sayah, and M.A.M. Gijs. 2001. Utilization of the sol-gel technique for the development of novel stationary phases for capillary electrochromatography on a chip. Sens. Actuators B 78:267-272.

69.Breadmore, M.C., S. Shrinivasan, K.A. Wolfe, M.E. Power, J.P. Ferrance, B. Hosticka, P.M Norris, and J.P. Landers. 2002. Towards a microchip-based chromatographic platform. Part 1 : evaluation of sol-gel phases for capillary electrochromatography. Electrophoresis 23:3487-3495.

70.Breadmore, M.C., S. Shrinivasan, J. Karlinsey, J.P. Ferrance, P.M. Norris, and J.P. Landers. 2003. Towards a microchip-based chromatographic platform. Part 2: sol-gel phases modified with polyelectrolyte multilayers for capillary electrochromatography. Electrophoresis 24:12611270

71.Jacobson, S.C., R. Hergenroder, L.B. Koutny, and J.M. Ramsey. 1994. Open-channel electrochromatography on a microchip. Anal. Chem. 66:2369-2373.

72.Oleschuk, R.D., L.L. Shultz-Lockyear, Y.B. Ning, and D.J. Harrison. 2000. Trapping of bead-based reagents within microfluidic systems on-chip solid-phase extraction and electrochro- 
matography. Anal. Chem. 72:585-590.

73.Ceriotti, L., N.F. de Rooij, and E. Verpoorte. 2002. An integrated fritless column for on-chip capillary electrochromatography with conventional stationary phases. Anal. Chem. 74:639647.

74.Slentz, B.E., N.A. Penner, and F.E. Regnier. 2002. Capillary electrochromatography of peptides on microfabricated poly(dimethylsiloxane) chips modified by cerium(IV)-catalyzed polymerization. J. Chromatog. A 948:225-233.

75.Effenhauser, C.S., A. Paulus, A. Manz, and H.M. Widmer. 1994. High-speed separation of antisense oligonucleotides on a micromachined capillary electrophoresis device. Anal. Chem. 66:2949-2953.

76.Lacher, N.A., N.F. de Rooij, E. Verpoorte, and S.M. Lunte. 2003. Comparison of the performance characteristics of poly(dimethylsiloxane) and Pyrex microchip electrophoresis devices for peptide separations. J. Chromatog. A 1004:225235

77.Culbertson, C.T., S.C. Jacobson, and J.M. Ramsey. 2000. Microchip devices for high-efficiency separations. Anal. Chem. 72:5814-5819.

78.Paegel, B.M., L.D. Hutt, P.C. Simpson, and R.A. Mathies. 2000. Turn geometry for minimizing band broadening in microfabricated capillary electrophoresis channels. Anal. Chem. 72:30303037.

79.Molho, J.I., A.E. Herr, B.P. Mosier, J.G. Santiago, T.W. Kenny, R.A. Brennen, G.B. Gordon, and B. Mohammadi. 2001. Optimization of turn geometries for microchip electrophoresis. Anal. Chem. 73:1350-1360.

80.Griffiths, S.K. and R.H. Nilson. 2001. Lowdispersion turns and junctions for microchannel systems. Anal. Chem. 73:272-278.

81.Kutter, J.P., S.C. Jacobson, and J.M. Ramsey. 1997. Integrated microchip device with electrokinetically controlled solvent mixing for isocratic and gradient elution in micellar electrokinetic chromatography. Anal. Chem. 69:5165-5171.

82.Hofmann, O., D.P. Che, K.A. Cruickshank, and U.R. Muller. 1999. Adaptation of capillary isoelectric focusing to microchannels on a glass chip. Anal. Chem. 71:678-686.

83.Mao, Q. and J. Pawliszyn. 1999. Demonstration of isoelectric focusing on an etched quartz chip with UV absorption imaging detection. Analyst 124:637-641.

84.Woolley, A.T. and R.A. Mathies. 1995. Ultrahigh-speed DNA-sequencing using capillary electrophoresis chips. Anal. Chem. 67:3676-3680.

85.Yao, S., D.S. Anex, W.B. Caldwell, D.W. Arnold, K.B. Smith, and P.G. Schultz. 1999. SDS capillary gel electrophoresis of proteins in microfabricated channels. Proc. Natl. Acad. Sci. USA 96:5372-5377.

86.Chen, X.X., H.K. Wu, C.D. Mao, and G.M. Whitesides. 2002. A prototype two-dimensional capillary electrophoresis system fabricated in poly(dimethylsiloxane). Anal. Chem. 74:17721778.

87.Woolley, A.T. and R.A. Mathies. 1994. Ultrahigh-speed DNA fragment separations using microfabricated capillary array electrophoresis chips. Proc. Natl. Acad. Sci. USA 91:1134811352

88.Landers, J.P. 2003. Molecular diagnostics on electrophoretic microchips. Anal. Chem. 75:2919-2927.
89.Ramsey, J.D., S.C. Jacobson, C.T. Culbertson, and J.M. Ramsey. 2003. High-efficiency, twodimensional separations of protein digests on microfluidic devices. Anal. Chem. 75:3758-3764.

90.Herr, A.E., J.I. Molho, K.A. Drouvalakis, J.C. Mikkelsen, P.J. Utz, J.G. Santiago, and T.W. Kenny. 2003. On-chip coupling of isoelectric focusing and free solution electrophoresis for multidimensional separations. Anal. Chem. 75:11801187.

91.Wu, Z.Y., N. Xanthopoulos, F. Reymond, J.S. Rossier, and H.H. Girault. 2002. Polymer microchips bonded by O-2-plasma activation. Electrophoresis 23:782-790.

92.Doherty, E.A.S., R.J. Meagher, M.N. Albarghouthi, and A.E. Barron. 2003. Microchannel wall coatings for protein separations by capillary and chip electrophoresis. Electrophoresis 24:3454.

93.Soper, S.A., A.C. Henry, B. Vaidya, M. Galloway, M. Wabuyele, and R.L. McCarley. 2002. Surface modification of polymer-based microfluidic devices. Anal. Chim. Acta 470:87-99.

94.Xiao, D.Q., T. Van Le, and M.J. Wirth. 2004. Surface modification of the channels of poly(dimethylsiloxane) microfluidic chips with polyacrylamide for fast electrophoretic separations of proteins. Anal. Chem. 76:2055-2061.

95.Hu, S.W., X.Q. Ren, M. Bachman, C.E. Sims, G.P. Li, and N.L. Allbritton. 2004. Surface-directed, graft polymerization within microfluidic channels. Anal. Chem. 76:1865-1870.

96.Schwarz, M.A. and P.C. Hauser. 2001. Recent developments in detection methods for microfabricated analytical devices. Lab Chip 1:1-6.

97.Lacher, N.A., K.E. Garrison, R.S. Martin, and S.M. Lunte. 2001. Microchip capillary electrophoresis/electrochemistry. Electrophoresis 22:2526-2536.

98.Wang, J. 2002. Electrochemical detection for microscale analytical systems: a review. Talanta 56:223-231.

99.Lion, N., T.C. Rohner, L. Dayon, I.L. Arnaud, E. Damoc, N. Youhnovski, Z.Y. Wu, C. Roussel, et al. 2003. Microfluidic systems in proteomics. Electrophoresis 24:3533-3562.

100. Verpoorte, E. 2003. Chip vision-optics for microchips. Lab Chip 3:42N-52N.

101.Thrush, E., O. Levi, W. Ha, K. Wang, S.J. Smith, and J.S. Harris. 2003. Integrated biofluorescence sensor. J. Chromatog. A 1013:103110 .

102.Chabinyc, M.L., D.T. Chiu, J.C. McDonald, A.D. Stroock, J.F. Christian, A.M. Karger, and G.M. Whitesides. 2001. An integrated fluorescence detection system in poly(dimethylsiloxane) for microfluidic applications. Anal. Chem. 73:4491-4498.

103.Ng, J.M.K., I. Gitlin, A.D. Stroock, and G.M. Whitesides. 2002. Components for integrated poly(dimethylsiloxane) microfluidic systems. Electrophoresis 23:3461-3473.

104.Effenhauser, C.S., G.J.M. Bruin, A. Paulus, and M. Ehrat. 1997. Integrated capillary electrophoresis on flexible silicone microdevices: analysis of DNA restriction fragments and detection of single DNA molecules on microchips. Anal. Chem. 69:3451-3457.

105.Fister, III, J.C., S.C. Jacobson, L.M. Davis, and J.M. Ramsey. 1998. Counting single chromophore molecules for ultrasensitive analysis and separations on microchip devices. Anal. Chem.
70:431-437

106.Foquet, M., J. Korlach, W.R. Zipfel, W.W. Webb, and H.G. Craighead. 2004. Focal volume confinement by submicrometer-sized fluidic channels. Anal. Chem. 76:1618-1626.

107.Jacobson, S.C., R. Hergenroder, A.W. Moore, Jr., and J.M. Ramsey. 1994. Precolumn reactions with electrophoretic analysis integrated on a microchip. Anal. Chem. 66:4127-4132.

108.Jin, L.J., B.C. Giordano, and J.P. Landers. 2001. Dynamic labeling during capillary or microchip electrophoresis for laser-induced fluorescence detection of protein-SDS complexes without pre- or postcolumn labeling. Anal. Chem. 73:4994-4999.

109.Jacobson, S.C., L.B. Koutny, R. Hergenroeder, A.W. Moore, Jr., and J.M. Ramsey. 1994 Microchip capillary electrophoresis with an integrated postcolumn reactor. Anal. Chem. 66:34723476.

110.Colyer, C.L., S.D. Mangru, and D.J. Harrison. 1997. Microchip-based capillary electrophoresis of human serum proteins. J. Chromatogr. A 781:271-276.

111.Liu, Y., R.S. Foote, S.C. Jacobson, R.S. Ramsey, and J.M. Ramsey. 2000. Electrophoretic separation of proteins on a microchip with noncovalent, postcolumn labeling. Anal. Chem. 72:4608-4613

112.Liang, Z., N. Chiem, G. Ocvirk, T. Tang, K. Fluri, and D.J. Harrison. 1996. Microfabrication of a planar absorbance and fluorescence cell for integrated capillary electrophoresis devices. Anal. Chem. 68:1040-1046.

113.Hawkins, K.R. and P. Yager. 2003. Nonlinear decrease of background fluorescence in polymer thin-films - a survey of materials and how they can complicate fluorescence detection in $\mathrm{mu}$ TAS. Lab Chip 3:248-252.

114.Lichtenberg, J., N.F. de Rooij, and E. Verpoorte. 2002. A microchip electrophoresis system with integrated in-plane electrodes for contactless conductivity detection. Electrophoresis 23:3769-3780.

115.Pumera, M., J. Wang, F. Opekar, I. Jelinek, J. Feldman, H. Lowe, and S. Hardt. 2002. Contactless conductivity detector for microchip capillary electrophoresis. Anal. Chem. 74:1968-1971.

116.Tanyanyiwa, J. and P.C. Hauser. 2002. Highvoltage capacitively coupled contactless conductivity detection for microchip capillary electrophoresis. Anal. Chem. 74:6378-6382.

117.Woolley, A.T., K. Lao, A.N. Glazer, and R.A. Mathies. 1998. Capillary electrophoresis chips with integrated electrochemical detection. Anal. Chem. 70:684-688.

118.Martin, R.S., A.J. Gawron, S.M. Lunte, and C.S. Henry. 2000. Dual-electrode electrochemical detection for poly(dimethylsiloxane)-fabricated capillary electrophoresis microchips. Anal Chem. 72:3196-3202.

119.Wang, J., B. Tian, and E. Sahlin. 1999. Micromachined electrophoresis chips with thick-film electrochemical detectors. Anal. Chem. 71:5436 5440.

120.Wang, J., B. Tian, and E. Sahlin. 1999. Integrated electrophoresis chips/amperometric detection with sputtered gold working electrodes. Anal Chem. 71:3901-3904.

121.Rossier, J.S., R. Ferrigno, and H.H. Girault. 2000. Electrophoresis with electrochemical detection in a polymer microdevice. J. Electroanal. 
Chem. 492:15-22.

122.Martin, R.S., K.L. Ratzlaff, B.H. Huynh, and S.M. Lunte. 2002. In-channel electrochemical detection for microchip capillary electrophoresis using an electrically isolated potentiostat. Anal. Chem. 74:1136-1143.

123.Gavin, P.F. and A.G. Ewing. 1997. Characterization of electrochemical array detection for continuous channel electrophoretic separations in micrometer and submicrometer channels. Anal. Chem. 69:3838-3845.

124.Deng, Y., J. Henion, J. Li, P. Thibault, C. Wang, and D.J. Harrison. 2001. Chip-based capillary electrophoresis/mass spectrometry determination of carnitines in human urine. Anal. Chem. 73:639-646.

125.Ramsey, R.S. and J.M. Ramsey. 1997. Generating electrospray from microchip devices using electroosmotic pumping. Anal. Chem. 69:11741178.

126.Xue, Q., F. Foret, Y.M. Dunayevskiy, P.M. Zavracky, N.E. McGruer, and B.L. Karger. 1997. Multichannel microchip electrospray mass spectrometry. Anal. Chem. 69:426-430.

127.Huikko, K., R. Kostiainen, and T. Kotiaho. 2003. Introduction to micro-analytical systems: bioanalytical and pharmaceutical applications. Eur. J. Pharm. Sci. 20:149-171.

128.Rohner, T.C., J.S. Rossier, and H.H. Girault. 2001. Polymer microspray with an integrated thick-film microelectrode. Anal. Chem. 73:53535357.

129.Gobry, V., J. van Oostrum, M. Martinelli, T.C. Rohner, F. Reymond, J.S. Rossier, and H.H. Girault. 2002. Microfabricated polymer injector for direct mass spectrometry coupling. Proteomics 2:405-412.

130.Zhang, B., H. Liu, B.L. Karger, and F. Foret. 1999. Microfabricated devices for capillary electrophoresis-electrospray mass spectrometry. Anal. Chem. 71:3258-3264.

131. Verpoorte, E. 2002. Microfluidic chips for clinical and forensic analysis. Electrophoresis 23:677712.

132.Hofgartner, W.T., A.F.R. Huhmer, J.P. Landers, and J.A. Kant. 1999. Rapid diagnosis of herpes simplex encephalitis using microchip electrophoresis of PCR products. Clin. Chem. 45:2120-2128

133.Emrich, C.A., H.J. Tian, I.L. Medintz, and R.A. Mathies. 2002. Microfabricated 384-lane capillary array electrophoresis bioanalyzer for ultrahigh-throughput genetic analysis. Anal. Chem. 74:5076-5083

134.Paegel, B.M., C.A. Emrich, G.J. Weyemayer, J.R. Scherer, and R.A. Mathies. 2002. High throughput DNA sequencing with a microfabricated 96-lane capillary array electrophoresis bioprocessor. Proc. Natl. Acad. Sci. USA 99:574579

135.Simpson, P.C., D. Roach, A.T. Woolley, T. Thorsen, R. Johnston, G.F. Sensabaugh, and R.A. Mathies. 1998. High-throughput genetic analysis using microfabricated 96-sample capillary array electrophoresis microplates. Proc. Natl. Acad. Sci. USA 95:2256-2261.

136.Kheterpal, I., J.R. Scherer, S.M. Clark, A. Radhakrishnan, J.Y. Ju, C.L. Ginther, G.F Sensabaugh, and R.A. Mathies. 1996. DNA sequencing using a four-color confocal fluorescence capillary array scanner. Electrophoresis 17:1852-1859.
137.Kricka, L.J. and P. Wilding. 2003. Microchip PCR. Anal. Bioanal. Chem. 377:820-825.

138.Wilding, P., M.A. Shoffner, and L.J. Kricka. 1994. PCR in a silicon microstructure. Clin. Chem. 40:1815-1818

139.Kopp, M.U., A.J. de Mello, and A. Manz. 1998. Chemical amplification: continuous-flow PCR on a chip. Science 280:1046-1048.

140.Woolley, A.T., D. Hadley, P. Landre, A.J. deMello, R.A. Mathies, and M.A. Northrup. 1996. Functional integration of PCR amplification and capillary electrophoresis in a microfabricated DNA analysis device. Anal. Chem. 68:40814086.

141.Burns, M.A., B.N. Johnson, S.N. Brahmasandra, K. Handique, J.R. Webster, M. Krishnan, T.S. Sammarco, P.M. Man, et al. 1998. An integrated nanoliter DNA analysis device. Science 282:484-487.

142.Waters, L.C., S.C. Jacobson, N. Kroutchinina, J. Khandurina, R.S. Foote, and J.M. Ramsey. 1998. Microchip device for cell lysis, multiplex PCR amplification, and electrophoretic sizing. Anal. Chem. 70:158-162.

143.Yuen, P.K., L.J. Kricka, P. Fortina, N.J. Panaro, T. Sakazume, and P. Wilding. 2001. Microchip module for blood sample preparation and nucleic acid amplification reactions. Genome Res. 11:405-412.

144.Liu, R.H., J.N. Yang, R. Lenigk, J. Bonanno, and P. Grodzinski. 2004. Self-contained, fully integrated biochip for sample preparation, polymerase chain reaction amplification, and DNA microarray detection. Anal. Chem. 76:18241831.

145.Liu, J., C. Hansen, and S.R. Quake. 2003. Solving the "world-to-chip" interface problem with a microfluidic matrix. Anal. Chem. 75:47184723

146.Hong, J.W., V. Studer, G. Hang, W.F. Anderson, and S.R. Quake. 2004. A nanoliter-scale nucleic acid processor with parallel architecture. Nat. Biotechnol. 22:435-439.

147.Koh, C.G., W. Tan, M.Q. Zhao, A.J. Ricco, and Z.H. Fan. 2003. Integrating polymerase chain reaction, valving, and electrophoresis in a plastic device for bacterial detection. Anal. Chem. 75:4591-4598.

148.Anderson, R.C., X. Su, G.J. Bogdan, and J. Fenton. 2000. A miniature integrated device for automated multistep genetic assays. Nucleic Acids Res. 28:e60.

149.Giordano, B.C., J. Ferrance, S. Swedberg, A.F.R. Huhmer, and J.P. Landers. 2001. Polymerase chain reaction in polymeric microchips: DNA amplification in less than 240 seconds. Anal. Biochem. 291:124-132.

150.Bousse, L., S. Mouradian, A. Minalla, H. Yee, K. Williams, and R. Dubrow. 2001. Protein sizing on a microchip. Anal. Chem. 73:1207-1212.

151.Gottschlich, N., S.C. Jacobson, C.T. Culbertson, and J.M. Ramsey. 2001. Two-dimensional electrochromatography/capillary electrophoresis on a microchip. Anal. Chem. 73:2669-2674.

152.Li, Y., J.S. Buch, F. Rosenberger, D.L. DeVoe, and C.S. Lee. 2004. Integration of isoelectric focusing with parallel sodium dodecyl sulfate gel electrophoresis for multidimensional protein separations in a plastic microfludic network. Anal. Chem. 76:742-748

153.Becker, H., K. Lowack, and A. Manz. 1998. Planar quartz chips with submicron channels for two-dimensional capillary electrophoresis applications. J. Micromech. Microeng. 8:24-28.

154.Griebel, A., S. Rund, F. Schonfeld, W. Dorner, R. Konrad, and S. Hardt. 2004. Integrated polymer chip for two-dimensional capillary gel electrophoresis. Lab Chip 4:18-23.

155.Andersson, H. and A. van den Berg. 2003. Microfluidic devices for cellomics: a review. Sens Actuators B 92:315-325.

156.Fu, A.Y., C. Spence, A. Scherer, F.H. Arnold and S.R. Quake. 1999. A microfabricated fluorescence-activated cell sorter. Nat. Biotechnol. 17:1109-1111.

157.Chiu, D.T., N.L. Jeon, S. Huang, R.S. Kane, C.J. Wargo, I.S. Choi, D.E. Ingber, and G.M. Whitesides. 2000. Patterned deposition of cells and proteins onto surfaces by using three-dimensional microfluidic systems. Proc. Natl. Acad Sci. USA 97:2408-2413.

158.Shelby, J.P., J. White, K. Ganesan, P.K. Rathod, and D.T. Chiu. 2003. A microfluidic model for single-cell capillary obstruction by Plasmodium falciparum infected erythrocytes. Proc. Natl. Acad. Sci. USA 100:14618-14622.

159.Wheeler, A.R., W.R. Throndset, R.J. Whelan, A.M. Leach, R.N. Zare, Y.H. Liao, K. Farrell, I.D. Manger, and A. Daridon. 2003. Microfluidic device for single-cell analysis. Anal. Chem. 75:3581-3586.

160.Blankenstein, G. and U.D. Larsen. 1998 Modular concept of a laboratory on a chip for chemical and biochemical analysis. Biosens. Bioelectron. 13:427-438.

161.Schrum, D.P., C.T. Culbertson, S.C. Jacobson, and J.M. Ramsey. 1999. Microchip flow cytometry using electrokinetic focusing. Anal. Chem. 71:4173-4177.

162.Fu, A.Y., H.P. Chou, C. Spence, F.H. Arnold and S.R. Quake. 2002. An integrated microfabricated cell sorter. Anal. Chem. 74:2451-2457.

163.Delamarche, E., A. Bernard, H. Schmid, B. Michel, and H. Biebuyck. 1997. Patterned delivery of immunoglobulins to surfaces using microfluidic networks. Science 276:779-781.

164.Chen, C.S., M. Mrksich, S. Huang, G.M. Whitesides, and D.E. Ingber. 1997. Geometric control of cell life and death. Science 276:1425 1428 .

165.Cokelet, G.R., R. Soave, G. Pugh, and L. Rathbun. 1993. Fabrication of in-vitro microvascular blood-flow systems by photolithography. Microvasc. Res. 46:394-400.

166.Wilding, P., J. Pfahler, H.H. Bau, J.N. Zemel, and L.J. Kricka. 1994. Manipulation and flow of biological-fluids in straight channels micromachined in silicon. Clin. Chem. 40:43-47.

167.Gifford, S.C., M.G. Frank, J. Derganc, C. Gabel, R.H. Austin, T. Yoshida, and M.W. Bitensky. 2003. Parallel microchannel-based measurements of individual erythrocyte areas and volumes. Biophys. J. 84:623-633.

Address correspondence to:

Daniel T. Chiu

Department of Chemistry

University of Washington

Seattle, WA 98195-1700, USA

e-mail: chiu@chem.washington.edu 